\title{
A Unified Framework for Hybrid Control: Model and Optimal Control Theory
}

\author{
Michael S. Branicky, Member, IEEE, Vivek S. Borkar, Senior Member, IEEE, and Sanjoy K. Mitter, Fellow
}

\begin{abstract}
Complex natural and engineered systems typically possess a hierarchical structure, characterized by continuousvariable dynamics at the lowest level and logical decision-making at the highest. Virtually all control systems today-from flight control to the factory floor-perform computer-coded checks and issue logical as well as continuous-variable control commands. The interaction of these different types of dynamics and information leads to a challenging set of "hybrid" control problems. We propose a very general framework that systematizes the notion of a hybrid system, combining differential equations and automata, governed by a hybrid controller that issues continuous-variable commands and makes logical decisions. We first identify the phenomena that arise in real-world hybrid systems. Then, we introduce a mathematical model of hybrid systems as interacting collections of dynamical systems, evolving on continuous-variable state spaces and subject to continuous controls and discrete transitions. The model captures the identified phenomena, subsumes previous models, yet retains enough structure on which to pose and solve meaningful control problems. We develop a theory for synthesizing hybrid controllers for hybrid plants in an optimal control framework. In particular, we demonstrate the existence of optimal (relaxed) and near-optimal (precise) controls and derive "generalized quasi-variational inequalities" that the associated value function satisfies. We summarize algorithms for solving these inequalities based on a generalized Bellman equation, impulse control, and linear programming.
\end{abstract}

Index Terms - Automata, control systems, differential equations, dynamic programming, hierarchical systems, hybrid systems, optimal control, state-space methods.

\section{INTRODUCTION}

$\mathbf{M}$ ANY COMPLICATED control systems today (e.g., those for flight control, manufacturing systems, and transportation) have vast amounts of computer code at their highest level. More pervasively, programmable logic controllers are widely used in industrial process control. We also see that today's products incorporate logical decision-making into even the simplest control loops (e.g., embedded systems). Thus, virtually all control systems today issue continuousvariable controls and perform logical checks that determine the mode - and hence the control algorithms - the continuous-

Manuscript received May 22, 1996; revised April 18, 1997. This work was supported by the Army Research Office and the Center for Intelligent Control Systems under Grants DAAL03-92-G-0164 and DAAL03-92-G-0115.

M. S. Branicky is with the Department of Electrical Engineering and Applied Physics, Case Western Reserve University, Cleveland, OH 441067221 USA (e-mail: branicky@alum.mit.edu).

V. S. Borkar is with the Department of Electrical Engineering, Indian Institute of Science, Bangalore 560012, India.

S. K. Mitter is with the Laboratory for Information and Decision Systems and Center for Intelligent Control Systems, Department of Electrical Engineering and Computer Science, Massachusetts Institute of Technology, Cambridge, MA 02139-4307 USA (e-mail: mitter@lids.mit.edu).

Publisher Item Identifier S 0018-9286(98)00925-8.

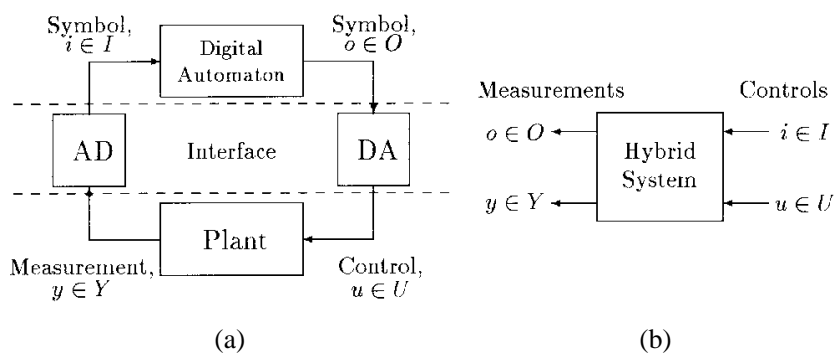

Fig. 1. (a) Hybrid system. (b) Hybrid control system.

variable system is operating under at any given moment. As such, these "hybrid control" systems offer a challenging set of problems.

Hybrid systems involve both continuous-valued and discrete-valued variables. Their evolution is given by equations of motion that generally depend on both. In turn these equations contain mixtures of logic and discrete-valued or digital dynamics and continuous-variable or analog dynamics. The continuous dynamics of such systems may be continuoustime, discrete-time, or mixed (sampled-data), but is generally given by differential equations. The discrete-variable dynamics of hybrid systems is generally governed by a digital automaton or input-output transition system with a countable number of states. The continuous and discrete dynamics interact at "event" or "trigger" times when the continuous state hits certain prescribed sets in the continuous state space; see Fig. 1(a).

Hybrid control systems are control systems that involve both continuous and discrete dynamics and continuous and discrete controls. The continuous dynamics of such a system is usually modeled by a controlled vector field or difference equation. Its hybrid nature is expressed by a dependence on some discrete phenomena, corresponding to discrete states, dynamics, and controls. The result is a system as in Fig. 1(b).

Examples of such systems are given in some depth in [1]. They include computer disk drives [2], transmissions and stepper motors [3], constrained robotic systems [4], and automated highway systems [5]. More generally, such systems arise whenever one mixes logical decision-making with the generation of continuous control laws. Thus, applications range from programmable logic controllers on our factory floors to flight vehicle management systems [6] in our skies.

So, "hybrid" systems are certainly pervasive today. But they have been with us at least since the days of the relay. Traditionally, though, the hybrid nature of systems and controllers has been suppressed by converting them into either purely discrete or purely continuous entities. The reason is that 
science and engineering's formal modeling, analysis, and control "toolboxes" deal largely—and largely successfully—with these "pure" systems.

It is no surprise, then, that there are two current paradigms for dealing with hybrid systems: aggregation and continuation. In the aggregation paradigm, one endeavors to treat the entire system as a finite automaton or discrete-event dynamic system (DEDS). This is usually accomplished by partitioning the continuous state space and considering only the aggregated dynamics from cell to cell in the partition (cf., [7]). In the continuation paradigm, one endeavors to treat the whole system as a differential equation. This is accomplished by 1) "simulating" or "embedding" the discrete actions in nonlinear ordinary differential equations (ODE's) or 2) treating the discrete actions as "disturbances" of some (usually linear) differential equation.

In current applications of interest (mentioned above), both these paradigms have been found lacking. In a nutshell, they are too conservative. Aggregation often leads to nondeterministic automata and yields the problem of how to pick appropriate partitions. Indeed, Digennaro et al. have shown that there are systems consisting of just two constant rate clocks with reset (evolving on the unit square $[0,1]^{2}$ and resetting to zero on hitting one) for which no partition exists that yields a deterministic finite automaton [8]. Continuation's first route hides the discrete dynamics in the right-hand sides of ODE's, yielding nonlinear systems for which there is a dearth of tools and engineering insight. Indeed, Branicky has shown that there are smooth, Lipschitz continuous ODE's in $\mathbb{R}^{3}$, which possess the power of universal computation, hence yielding most control questions in $\mathbb{R}^{3}$ undecidable [9] (one such question is constructed in Section IX-B). Continuation's second route may treat the discrete dynamics as small unmodeled dynamics (and then use robust control), slowly-varying (and gain-scheduling), or rare and independent of the continuous state (jump linear systems). In hybrid systems of interest, each or all of these assumptions may be violated, leading to hopelessly conservative designs.

Herein, we propose a truly hybrid paradigm for hybrid systems by developing a new, unified framework that captures both the important discrete and continuous features of such systems - and their interactions - in such a way that we can build on the considerable engineering insight on both sides and provide natural, nonconservative solutions to hybrid control problems. In particular, in this paper we address and answer the problem of synthesizing hybrid controllers-which issue continuous controls and make discrete decisions - that achieve certain prescribed safety and performance goals for hybrid systems.

Problem 1.1: How do we control a plant as in Fig. 1(b) with a controller as in Fig. 1(b)?

In order to turn this profound, abstract problem into a tractable one, we require two prerequisites:

P1) a mathematical model for a box like Fig. 1(b);

P2) a mathematical control problem which leads to a hybrid controller.
Briefly, we build on the structure of dynamical systems for P1) and use an optimal control framework for P2). The details follow.

In other work, we have looked at real-world examples and previously posed hybrid systems models and identified four phenomena that need to be covered by any useful model: 1) autonomous switching; 2) autonomous impulses; 3) controlled switching; and 4) controlled impulses. In [1], Branicky introduced general hybrid dynamical systems (GHDS's) as interacting collections of dynamical systems, each evolving on continuous-variable state spaces, with switching among systems occurring at "autonomous jump times" when the state variable intersects specified subsets of the constituent state spaces. Controlled GHDS's, or CGHDS's, first add the possibility of continuous controls for each constituent dynamical system. They also allow discrete decisions at autonomous jump times as well the ability to discontinuously reset state variables at "intervention times" when the state satisfies certain conditions, given by its membership in another collection of specified subsets of the state space. In general, the allowed resettings depend on the state.

The CGHDS model has three important properties as follows. It covers the identified phenomena, encompasses all the studied previous models, and has sufficient mathematical structure to allow the posing and proving of deeper results [1]. This satisfies P1).

For P2), we use a variant of the CGHDS that possesses all its generalization and structural properties and covers most situations of interest to both control engineers and computer scientists. It also includes conventional impulse control [10]. Because of this, we dubbed it the "unified model." Finally, we use an optimal control framework to formulate and solve for hybrid controllers governing hybrid plants. In particular, our collection is indexed by $i \in \mathbb{Z}_{+}$, and our dynamical systems are given by controlled vector fields in $\mathbb{R}^{d_{i}}$, for some $d_{i} \in \mathbb{Z}_{+}$. Maps representing the costs of continuous controls and autonomous and controlled jumps are presumed. The control objective is then to minimize the total accumulated cost over all available decisions and controls.

The paper is organized as follows. In the next section, we quickly review previous work on hybrid control. In Section III we 1) identify the phenomena present in real-world systems we must capture and 2) classify previous modeling efforts. In Section IV, we present our CGHDS model and show that it is sufficiently rich to cover the identified phenomena and reviewed models. In Section V, we define an optimal control problem on our unified model. The problem, and all assumptions used in obtaining the remaining results, are expressly stated. Sections VI and VII contain the main theoretical results, which are as follows.

- We prove the existence of optimal (relaxed or chattering) controls and near-optimal (precise or nonchattering) controls.

- We derive "generalized quasi-variational inequalities" (GQVI's) that the associated value function is expected to satisfy. 
Further, the necessity of our assumptions-or ones like them-is demonstrated. Section VIII gives some quick examples, and in Section IX are conclusions and a discussion, including open issues and a summary of our work to date on control synthesis algorithms. The latter is based on solving our GQVI's and will appear in full as a future paper.

The optimal control theory of this paper grew out of [11]. Early references are [12]-[14].

Below, $\mathbb{R}, \mathbb{R}_{+}, \mathbb{Z}$, and $\mathbb{Z}_{+}$denote the reals, nonnegative reals, integers, and nonnegative integers, respectively. $X \backslash U$ represents the complement of $U$ in $X ; \bar{U}$ represents the closure of $U, U^{\circ}$ its interior, $\partial U$ its boundary; $C(X, Y)$ denotes the space of continuous functions with domain $X$ and range $Y$; $v^{T}$ denotes the transpose of vector $v$; and $\|x\|$ denotes an arbitrary norm of vector $x$. More special notation is defined as it is introduced.

\section{PREVIOUS WORK}

Hybrid systems are certainly pervasive today, but they have been with us at least since the days of the relay. The earliest direct reference we know of is the visionary work of Witsenhausen from MIT, who formulated a class of hybrid-state continuous-time dynamic systems and examined an optimal control problem [15]. Another early gem is the modeling paper of Tavernini [16].

Hybrid systems is now a rapidly expanding field that has just started to be addressed more wholeheartedly by the control and computer science communities. Explicit reference to general papers is beyond our scope here (see [1] for review, references, and other results). However, our modeling work has been influenced by [2]-[4], [12], and [15]-[17].

Our work was largely inspired by the well-known theories of impulse control and piecewise deterministic processes [18]-[21]. Close to our results are those of [22], discovered after this work was completed. That paper considers switching and "impulse obstacle" operators akin to those in (13) and (12) for autonomous and (controlled) impulsive jumps, respectively. Yong restricts the switching and impulse operators to be uniform in the whole space, which is unrealistic in hybrid systems. However, he derives viscosity solutions of his corresponding Hamilton-Jacobi-Bellman system. His work may be useful in deriving viscosity solutions to our GQVI's.

Also after this work was completed, we became aware of the model and work of [15], mentioned above. In that paper, Witsenhausen considers an optimal terminal constraint problem on his hybrid systems model. His model contains no autonomous impulses, no controlled switching, and no controlled impulses.

Optimal control of hybrid systems has also been considered in [23] (for the discrete-time case) and [17]. Kohn is the first we know of to speak of using relaxed controls and their $\epsilon$-optimal approximations in a hybrid systems setting (see the discussion and references of [17, Appendix I]). The algorithmic importance of these was further described in [24]. A different approach to the control of hybrid systems has been pursued by Kohn and Nerode [17, Appendix II], in which the discrete portion of the dynamics is itself designed as a

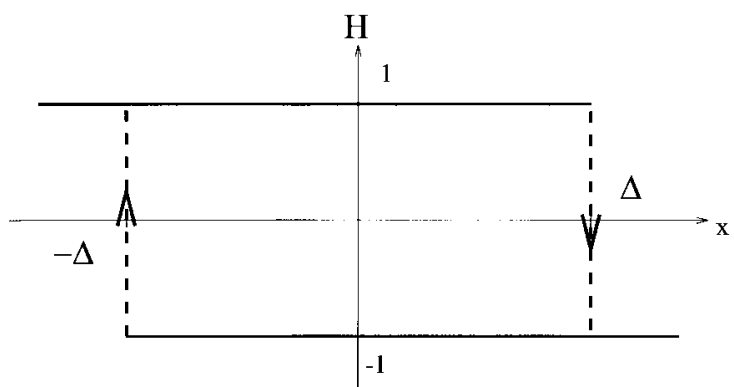

Fig. 2. Hysteresis function.

realizable implementation (i.e., a sufficient approximation) of some continuous controller. Finally, viable control of hybrid systems has been considered by researchers subsequent to our initial findings [25], [26].

\section{A TAXONOMY FOR HYBRID SYSTEMS}

\section{A. Hybrid Phenomena}

A hybrid system has continuous dynamics modeled by a differential equation

$$
\dot{x}(t)=\xi(t), \quad t \geq 0
$$

that depends on some discrete phenomena. Here, $x(t)$ is the continuous component of the state taking values in some subset of a Euclidean space. $\xi(t)$ is a controlled vector field that generally depends on $x(t)$, the continuous component $u(t)$ of the control policy, and the aforementioned discrete phenomena.

An examination of real-world examples and a review of other hybrid systems models has led us to an identification of these phenomena. The discrete phenomena generally considered are as follows. The real-world examples we examined may be found in [1] and [12].

1) Autonomous Switching: Here the vector field $\xi(\cdot)$ changes discontinuously, or "switches," when the state $x(\cdot)$ hits certain "boundaries" [16], [17]. The simplest example of this is when it changes depending on a "clock" that may be modeled as a supplementary state variable [3].

Example 3.1-Hysteresis: Consider a control system with hysteresis

$$
\dot{x}=f(x, u) \equiv H(x)+u
$$

where the multivalued function $H$ is shown in Fig. 2.

Note: This system is not just a differential equation whose right-hand side is piecewise continuous. There is "memory" in the system, which affects the value of the vector field. Indeed, such a system naturally has a finite automaton associated with the hysteresis function $H$, as pictured in Fig. 3.

2) Autonomous Impulses: Here the continuous state $x(\cdot)$ changes impulsively on hitting prescribed regions of the state space [4], [27]. The simplest examples possessing this phenomenon are those involving collisions. 


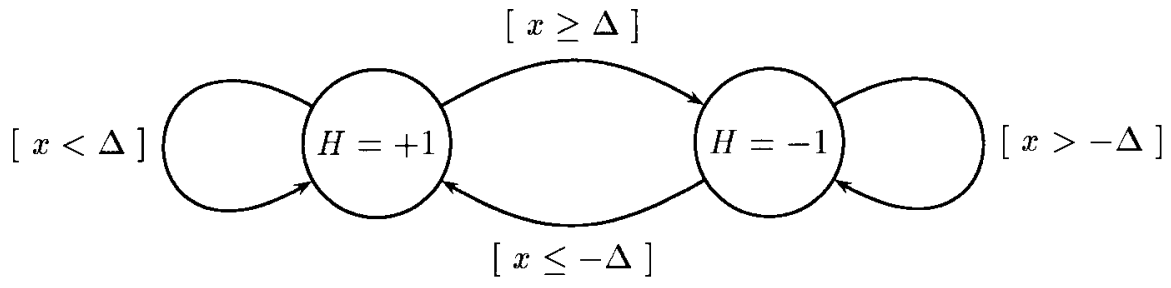

Fig. 3. Finite automaton associated with hysteresis function.

Example 3.2-Collisions: Consider the case of the vertical and horizontal motion of a ball of mass $m$ in a room under gravity with constant $g$. In this case, the dynamics are given by

$$
\begin{array}{ll}
\dot{x}=v_{x}, & \dot{v}_{x}=0 \\
\dot{y}=v_{y}, & \dot{v}_{y}=-m g .
\end{array}
$$

Further, upon hitting the boundaries $\{(x, y) \mid y=0$ or $y=C\}$, we instantly set $v_{y}$ to $-\rho v_{y}$, where $\rho \in[0,1]$ is the coefficient of restitution. Likewise, upon hitting $\{(x, y) \mid x=0$ or $x=R\}$ $v_{x}$ is set to $-\rho v_{x}$.

3) Controlled Switching: Here $\xi(\cdot)$ switches in response to a control command with an associated cost. This can be interpreted as switching between different vector fields [21]. Controlled switching arises, for instance, when one is allowed to pick among a number of vector fields $\dot{x}=f_{i}(x)$, $i \in\{1,2, \cdots, N\}$.

Example 3.3-Satellite Control: As a simple example of satellite control consider

$$
\ddot{\theta}=\tau_{\text {eff }} v
$$

where $\theta$ is angular position, $\dot{\theta}$ angular velocity of the satellite, and $v \in\{-1,0,1\}$, depending on whether the reaction jets are full reverse, off, or full on.

An example that includes controlled switching and continuous controls is the following.

Example 3.4-Transmission: Consider a simplified manual transmission model, modified from one in [3]

$$
\begin{aligned}
& \dot{x}_{1}=x_{2} \\
& \dot{x}_{2}=\left[-a\left(x_{2} / v\right)+u\right] /(1+v)
\end{aligned}
$$

where $x_{1}$ is the ground speed, $x_{2}$ is the engine RPM, $u \in[0,1]$ is the throttle position, and $v \in\{1,2,3,4\}$ is the gear shift position. The function $a$ is positive for positive argument.

4) Controlled Impulses: Here $x(\cdot)$ jumps in response to a control command with an associated cost [10].

Example 3.5-Inventory Management: In a simple inventory management model [10], there is a "discrete" set of restocking times $\theta_{1}<\theta_{2}<\cdots$ as well as associated order amounts $\alpha_{1}, \alpha_{2}, \cdots$. The equations governing the stock at any given moment are

$$
\dot{y}(t)=-\mu(t)+\sum_{i} \delta\left(t-\theta_{1}\right) \alpha_{i}
$$

where $\mu$ represents degradation or utilization dynamics and $\delta$ is the Dirac delta function.

\section{B. Classification of Hybrid Systems Models}

In this section, we give explicit representations of the broad classes of hybrid systems for which our theory and algorithms are applicable.

A (continuous-time) autonomous-switching hybrid system may be defined as follows:

$$
\begin{aligned}
\dot{x}(t) & =f(x(t), q(t)) \\
q^{+}(t) & =\nu(x(t), q(t))
\end{aligned}
$$

where $x(t) \in \mathbb{R}^{n}, q(t) \in Q \simeq\{1, \cdots, N\}$. Here, $f(\cdot, q): \mathbb{R}^{n} \rightarrow \mathbb{R}^{n}, q \in Q$, each globally Lipschitz continuous is the continuous dynamics of (2); and $\nu: \mathbb{R}^{n} \times Q \rightarrow Q$ represents its finite dynamics.

Note: The notation $t^{-}$may be used to indicate that the finite state is piecewise continuous from the right: $q(t)=$ $\nu\left(x(t), q\left(t^{-}\right)\right)$. Likewise, $q\left(t^{+}\right)=\nu(x(t), q(t))$ denotes it is piecewise-continuous from the left. To avoid making the distinction here, we have used Sontag's more evocative discretetime transition notation, where $q^{+}(t)$ is used to denote the "successor" of $q(t)$. Its "predecessor" is denoted $q^{-}(t)$. This notation makes sense since no matter which convention is used for $q(t)$ 's piecewise continuity, we still have $q^{+}(t)=q\left(t^{+}\right)$.

Thus, starting at $\left[x_{0}, i\right]$, the continuous-state trajectory $x(\cdot)$ evolves according to $\dot{x}=f(x, i)$. If $x(\cdot)$ hits some $(\nu(\cdot, i))^{-1}(j)$ at time $t_{1}$, then the state becomes $\left[x\left(t_{1}\right), j\right]$, from which the process continues. Clearly, this is an instantiation of autonomous switching. Switchings that are a fixed function of time may be taken care of by adding another state dimension, as usual. Examples are the Tavernini model [16] and the autonomous version of Witsenhausen's model [15].

By a continuous-controlled autonomous-switching hybrid system we have in mind a system of the form

$$
\begin{aligned}
\dot{x}(t) & =f(x(t), q(t), u(t)) \\
q^{+}(t) & =\nu(x(t), q(t), u(t))
\end{aligned}
$$

where everything is as above except that $u(t) \in \mathbb{R}^{m}$, with $f$ and $\nu$ modified appropriately. An example is Witsenhausen's model [15]. An autonomous-impulse hybrid system is a system

$$
\begin{aligned}
\dot{x}(t) & =f(x(t)), & & x(t) \notin M \\
x^{+}(t) & =J(x(t)), & & x(t) \in M
\end{aligned}
$$

where $x(t) \in \mathbb{R}^{n}, J: \mathbb{R}^{n} \rightarrow \mathbb{R}^{n}$, and $M \subset \mathbb{R}^{n}$. Examples include autonomous systems with impulse effect [27].

Finally, a hybrid system with autonomous switching and autonomous impulses is just a combination of (2) and (4). 


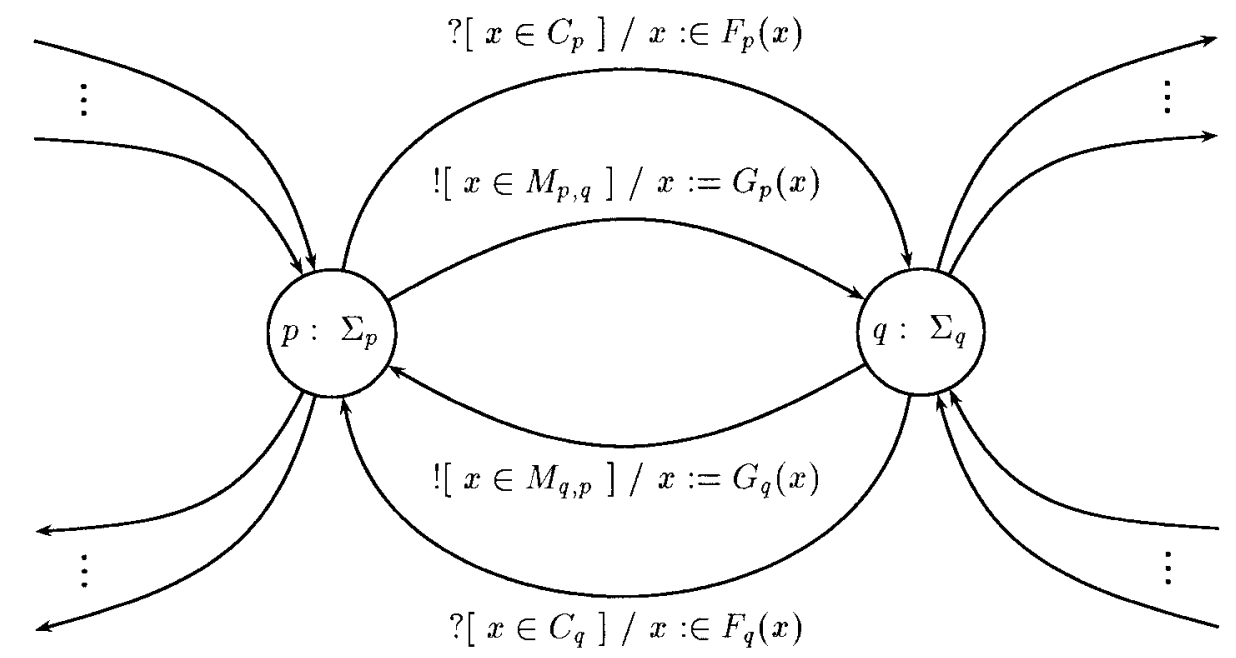

Fig. 4. Automaton associated with CGHDS.

Examples include the model of Back et al. [4] and hence all the autonomous models in [3], [15]-[17], and [28] (see [1] and [12]). Likewise, we can define discrete-time autonomous and controlled hybrid systems by replacing the ODE's above with difference equations. In this case, (2) represents a simplified view of some of the models in [3]. Also, adding controls-both discrete and continuous-is straightforward. Finally, nonuniform continuous state spaces, i.e., $x(t) \in X_{q(t)}$, may be added with little change.

The thesis [1] offers an in-depth review of previous hybrid systems models, including comparisons, and a more complete taxonomy for hybrid systems that is inclusive of the foregoing.

\section{HYBRID DYNAMICAL SYSTEMS}

\section{A. Mathematical Model}

The notion of a "dynamical system" has a long history as an important conceptual tool in science and engineering [29]-[34]. It is the foundation of our formulation of hybrid dynamical systems.

Briefly, a dynamical system is a system $\Sigma=[X, \Gamma, \phi]$, where $X$ is an arbitrary topological space, the state space of $\Sigma$. The transition semigroup $\Gamma$ is a topological semigroup with identity. The (extended) transition map $\phi: X \times \Gamma \rightarrow X$ is a continuous function satisfying the identity and semigroup properties [34].

Examples of dynamical systems abound, including autonomous ODE's, autonomous difference equations, finite automata, pushdown automata, Turing machines (TM's), Petri nets, etc. As seen from these examples, both digital and analog systems can be viewed in this formalism. The utility of this has been noted since the earliest days of control theory [32], [33].

We will also denote by dynamical system the system $\Sigma=$ $[X, \Gamma, f]$, where $X$ and $\Gamma$ are as above, but the transition function $f$ is the generator of the extended transition function $\phi$.

Briefly, a hybrid dynamical system is an indexed collection of dynamical systems along with some map for "jumping" among them (switching dynamical system and/or resetting the state). This jumping occurs whenever the state satisfies certain conditions, given by its membership in a specified subset of the state space. Hence, the entire system can be thought of as a sequential patching together of dynamical systems with initial and final states, the jumps performing a reset to a (generally different) initial state of a (generally different) dynamical system whenever a final state is reached.

Formally, a controlled general hybrid dynamical system (CGHDS) [1] is a system

$$
H_{c}=[Q, \Sigma, \mathbf{A}, \mathbf{G}, \mathbf{V}, \mathbf{C}, \mathbf{F}]
$$

with constituent parts as follows.

- $Q$ is the set of index states or discrete states.

- $\boldsymbol{\Sigma}=\left\{\Sigma_{q}\right\}_{q \in Q}$ is the collection of controlled dynamical systems, where each $\Sigma_{q}=\left[X_{q}, \Gamma_{q}, f_{q}, U_{q}\right]$ (or $\Sigma_{q}=$ $\left.\left[X_{q}, \Gamma_{q}, \phi_{q}, U_{q}\right]\right)$ is a controlled dynamical system. Here, the $X_{q}$ are the continuous state spaces and $\phi_{q}$ (or $f_{q}$ ) are the continuous dynamics; $U_{q}$ is the set of continuous controls.

- $\mathbf{A}=\left\{A_{q}\right\}_{q \in Q}, A_{q} \subset X_{q}$ for each $q \in Q$, is the collection of autonomous jump sets.

- $\mathbf{G}=\left\{G_{q}\right\}_{q \in Q}$, where $G_{q}: A_{q} \times V_{q} \rightarrow S$ is the autonomous jump transition map, parameterized by the transition control set $V_{q}$, a subset of the collection $\mathbf{V}=$ $\left\{V_{q}\right\}_{q \in Q}$; they are said to represent the discrete dynamics and controls.

- $\mathbf{C}=\left\{C_{q}\right\}_{q \in Q}, C_{q} \subset X_{q}$, is the collection of controlled jump sets.

- $\mathbf{F}=\left\{F_{q}\right\}_{q \in Q}$, where $F_{q}: C_{q} \rightarrow 2^{S}$ is the collection of controlled jump destination maps.

Thus, $S=\bigcup_{q \in Q} X_{q} \times\{q\}$ is the hybrid state space of $H$. The case where the sets $U_{q}$ and $\mathbf{V}$ through $\mathbf{F}$ above are empty is simply a GHDS: $H=[Q, \mathbf{\Sigma}, \mathbf{A}, \mathbf{G}]$.

A CGHDS can be pictured as an automaton as in Fig. 4. There, each node is a constituent dynamical system, with the index the name of the node. Each edge represents a possible transition between constituent systems, labeled by the appropriate condition for the transition's being "enabled" and 


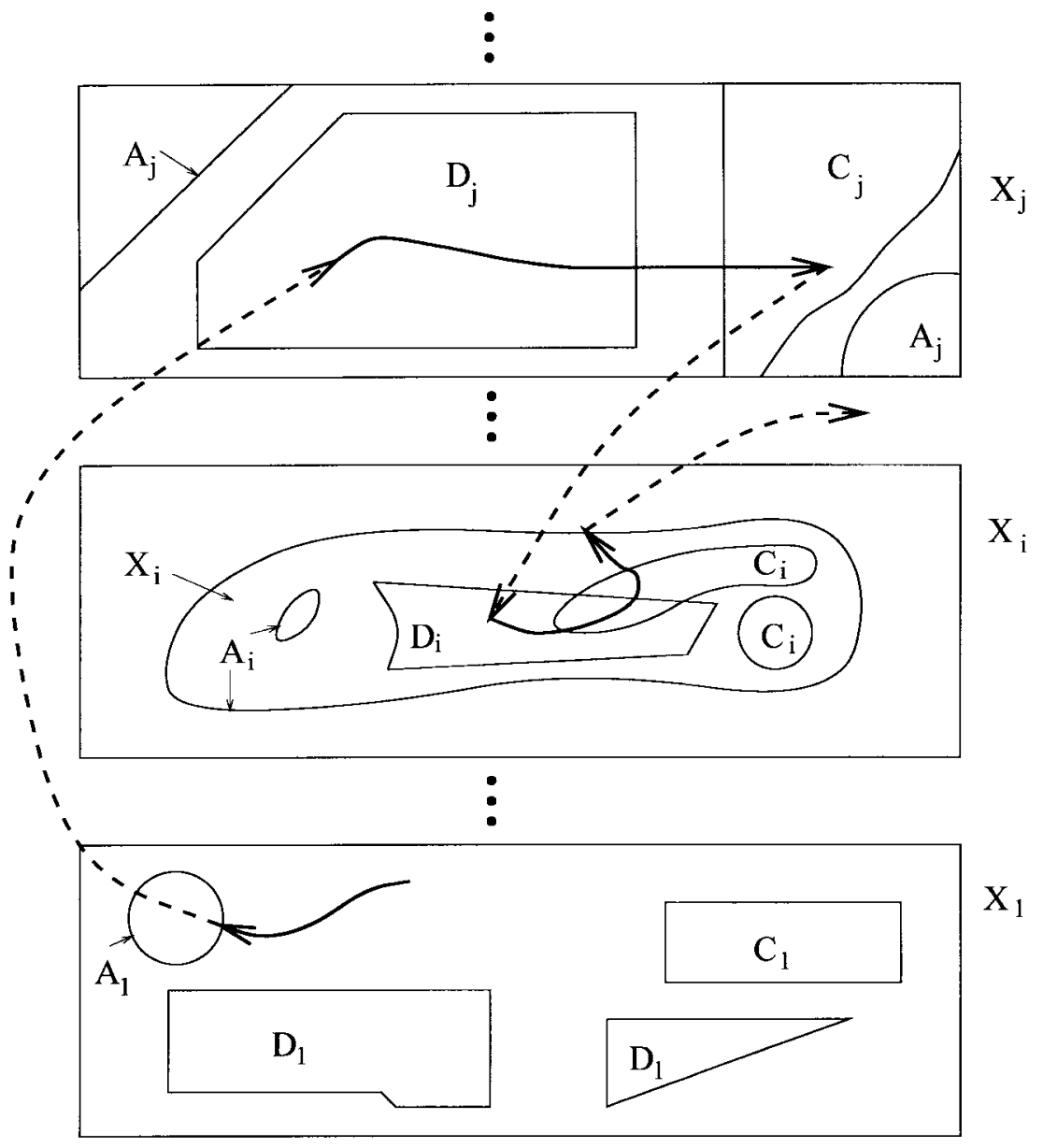

Fig. 5. Example dynamics of a CGHDS.

the update of the continuous state (cf., [35]). The notation ![condition] denotes that the transition must be taken when enabled. The notation ?[condition] denotes an enabled transition that may be taken on command; ": $\in$ " means reassignment to some value in the given set.

Roughly, the dynamics of $H_{c}$ are as follows. ${ }^{1}$ The system is assumed to start in some hybrid state in $S \backslash A$, say $s_{0}=$ $\left(x_{0}, q_{0}\right)$. It evolves according to $\phi_{q_{0}}(\cdot, \cdot, u)$ until the state enters-if ever-either $A_{q_{0}}$ or $C_{q_{0}}$ at the point $s_{1}^{-}=\left(x_{1}^{-}, q_{0}\right)$. If it enters $A_{q_{0}}$, then it must be transferred according to transition map $G_{q_{0}}\left(x_{1}^{-}, v\right)$ for some chosen $v \in V_{q_{0}}$. If it enters $C_{q_{0}}$, then we may choose to jump and, if so, we may choose the destination to be any point in $F_{q_{0}}\left(x_{1}^{-}\right)$. Either way, we arrive at a point $s_{1}=\left(x_{1}, q_{1}\right)$ from which the process continues; see Fig. 5.

\section{B. Notes}

The following are some important notes about CGHDS's.

1) Dynamical Systems: GHDS with $|Q|=1$ and A empty recover all these.

2) Hybrid Systems: The case of a GHDS with $|Q|$ finite, where each $X_{q}$ is a subset of $\mathbb{R}^{n}$ and each $\Gamma_{q}=\mathbb{R}$ largely corresponds to the usual notion of a hybrid system, viz, a

\footnotetext{
${ }^{1}$ Precise statements appear in [1, Sec. 4.3].
}

coupling of finite automata and differential equations [9], [12], [36]. Herein, a hybrid system is a GHDS with $Q$ countable and with $\Gamma_{q} \equiv \mathbb{R}$ (or $\mathbb{R}_{+}$) and $X_{q} \subset \mathbb{R}^{d_{q}}, d_{q} \in \mathbb{Z}_{+}$, for all $q \in Q:\left[Q,\left[\left\{X_{q}\right\}_{q \in Q}, \mathbb{R}_{+},\left\{f_{q}\right\}_{q \in Q}, \mathbf{A}, \mathbf{G}\right]\right.$, where $f_{q}$ is a vector field on $X_{q} \subset \mathbb{R}^{d_{q}}$.

3) Changing State Space: The state space may change. This is useful in modeling component failures or changes in dynamical description based on autonomous or controlled events which change it. Examples include the collision of two inelastic particles or an aircraft mode transition that changes variables to be controlled [38]. We also allow the $X_{q}$ to overlap and the inclusion of multiple copies of the same space. This may be used, for example, to take into account overlapping local coordinate systems on a manifold [4].

4) Refinements: We may refine the concept of a CGHDS by adding

- outputs, including state-output for each constituent system as for dynamical systems [1], [34] and edge-output: $\mathbf{O}=$ $\left\{O_{q}\right\}_{q \in Q}, \eta=\left\{\eta_{q}\right\}_{q \in Q}$, where $\eta_{q}: A_{q} \rightarrow O_{q}$ produces an output at each jump time.

\footnotetext{
${ }^{2}$ Here, we may take the view that the system evolves on the state space $\mathbb{R}^{*} \times Q$, where $\mathbb{R}^{*}$ denotes the set of finite, but variable-length real-valued vectors. For example, $Q$ may be the set of labels of a computer program and $x \in \mathbb{R}^{*}$ the values of all currently allocated variables. This then includes Smale's tame machines [37].
} 
- $\Delta: A_{q} \rightarrow \mathbb{R}_{+}$, the jump delay map, which can be used to account for the time which abstracted-away lower-level transition dynamics actually take. ${ }^{3}$

- We may add a transition time map or timing map $\tau: X \times$ $\Gamma \rightarrow \mathbb{R}$ (or $\mathbb{R}_{+}$) for some or all dynamical systems. Of particular interest are maps where $\tau$ is constant on $X$ and those that, in addition, are homomorphisms with subsets of $\mathbb{R}\left(\mathbb{R}_{+}\right)$. In the generator case, the timing map can be defined on $X \times \Gamma_{g}$.

Timing maps provide a mechanism for reconciling different "time scales" by giving a uniform meaning to different transition semigroups in a hybrid system $[1, \mathrm{Sec}$. IV].

- Marked states (including initial, final, or accepting states).

Other Notes:

1) Nondeterminism in transitions may be taken care of by partitioning [condition] into those which are controlled and uncontrolled (cf., DEDS). Disturbances (and other nondeterminism) may be modeled by partitioning $U, V$, and $C$ into portions that are under the influence of the controller or nature, respectively. Systems with stateoutput, edge-output, and autonomous and controlled jump delay maps ( $\Delta_{a}$ and $\Delta_{c}$, respectively) may be added as above.

2) The model includes the "unified" model posed by Branicky et al. [12] that is used below. It thus includes several other previously posed hybrid systems models [3], [4], [15]-[17], [28]. It also includes systems with impulse effect [27] and hybrid automata [39].

\section{Inclusion of Discrete Phenomena and Previous Models}

We now show how CGHDS encompasses the discrete phenomenon of Section III-A, and how it subsumes the hybrid systems models classified above.

First, we have a simplification. If a set of parameters or controls is countable and discrete, such as a set of strings, we may take it to be isomorphic with a subset of $\mathbb{Z}_{+}$. On the other hand, consider a set of parameters or controls $U$, where $U$ is a compact, connected, locally connected metric space $U$. By the Hahn-Mazurkiewicz theorem [40], $U$ is the continuous image of $[0,1]$ under some map, and thus we may set $U=[0,1]$ without any loss of generality. Thus, we may assume below without any loss of generality that parameters and controls take values in a subset $P \subset \mathbb{R}^{m}$.

1) Autonomous Switching and Impulses: These are clearly taken care of with the sets $A_{i}$.

2) Controlled Switching and Impulses: These are clearly taken care of with the sets $C_{i}$.

Remark 4.1: Autonomous (respectively, controlled) switching can be viewed as a special case of autonomous (respectively, controlled) jumps.

Proof: See the Appendix.

\footnotetext{
${ }^{3}$ Think of modeling the closure time of a discretely controlled hydraulic valve or trading imperfections in economic markets. This mechanism shows how hybrid systems naturally arise in hierarchical systems as one moves up different levels of abstraction.
}

3) Digital Automata: A variety of automata interacting with continuous dynamics are automatically subsumed by the discrete dynamics of our model. The automata structure may be captured by examining them; see Fig. 4. For example, if that picture had a finite number of nodes, each with a single nonempty edge emanating from it, the resulting automaton would be a deterministic finite automaton.

4) Other Hybrid Models: Our model subsumes all those classified and referenced in Section III-B. For example, models of the form of (2) can be accommodated merely by taking $Q \equiv Q$ and $f_{q}(\cdot) \equiv f(\cdot, q)$. The other classified models follow readily [12].

Example 4.2-Brockett's Type-D Hybrid System: In [3], Brockett introduces a type-D hybrid system as follows:

$$
\begin{aligned}
\dot{x}(t) & =f(x(t), u(t), z\lfloor p\rfloor) \\
\dot{p}(t) & =r(x(t), u(t), z\lfloor p\rfloor) \\
z\lceil p\rceil & =\nu(x[t], z\lfloor p\rfloor, v\lfloor p\rfloor)
\end{aligned}
$$

where $x(t) \in X \subset \mathbb{R}^{n}, u(t) \in U \subset \mathbb{R}^{m}, p(t) \in \mathbb{R}, v\lfloor p\rfloor \in$ $V, z \in Z, f: \mathbb{R}^{n} \times \mathbb{R}^{m} \times Z \rightarrow \mathbb{R}^{n}, r: \mathbb{R}^{n} \times \mathbb{R}^{m} \times Z \rightarrow \mathbb{R}$, and $\nu: \mathbb{R}^{n} \times Z \times V \rightarrow Z$. Here, $X$ and $U$ are open subsets of $\mathbb{R}^{n}$ and $\mathbb{R}^{m}$, respectively, and $V$ and $Z$ are isomorphic to subsets of $\mathbb{Z}_{+}$. Finally, $\lfloor x\rfloor$ denotes the greatest integer less than or equal to $x$, and, in an abuse of common notation, $\lceil x\rceil$ denotes the least integer greater than $x$.

Brockett's $D$ model may be captured by ours by choosing $Q=Z$, and for each $z \in Q$, defining $U_{z}=U, V_{z}=V$, $X_{z}=X \times[0,1]$ (with dimensions representing $x$ and $p-\lfloor p\rfloor$ )

$$
f_{z}=\left[\begin{array}{l}
f(\cdot, \cdot, z) \\
r(\cdot, \cdot, z)
\end{array}\right]
$$

$A_{z}=X \times\{1\}$, and $G_{z}((x, 1), v)=((x, 0), \nu(x, z, v))$.

It is clear that this can be extended to include the other models in [3]. Subsumption of the other referenced models follow readily [12]. ${ }^{4}$

5) Setting Parameters and Timers: A system which, upon hitting boundaries, sets parameters from an arbitrary compact set $P \subset \mathbb{R}^{p}$ can be modeled in our framework by redefining $X_{i}^{\prime}=X_{i} \times \mathbb{R}^{p}$, and $V^{\prime}=V \times P$, and defining $f_{i}^{\prime}: X_{i}^{\prime} \times U \rightarrow$ $\mathbb{R}^{d_{i}} \times \mathbb{R}^{p}$ as

$$
f_{i}^{\prime}((x, p), u)=\left[f_{i}(x, u), 0\right]^{T}
$$

and $G^{\prime}: A \times P \times V \times P \rightarrow X_{i} \times P$ as

$$
G^{\prime}\left(x, p, v, p^{\prime}\right)=\left[G(x, v), p^{\prime}\right]^{T}
$$

each for all possible arguments. A system which sets timers upon hitting boundaries can be modeled by a vector of the rate equations as in the vector form of Brockett's $D$ model

\footnotetext{
${ }^{4}$ Herein, $A$ subsumes $B$ means that every system described by the equations of model $B$ can be described by the equations of model $A$. From the original papers, it is clear that the referenced models were developed for a variety of purposes. Moreover, there is a direct tradeoff between the generality of a model and what one can prove about such a model. Therefore, "subsumption" of one model in another does not reflect any bias of the more general model's being "superior." Indeed, below, we restrict the class of CGHDS in forming our "unified model." That model is still abstract enough to capture the collected hybrid phenomena and subsume the models referenced here. However, the restrictions on it will allow us to solve optimal control problems.
} 
of hybrid systems, which in turn can be modeled in our framework as previously discussed.

\section{The Control Problem}

\section{A. Unified Model}

Now, we come to our unified model for hybrid control. Though a restriction of our CGHDS it still encompasses all identified phenomena and previously posed models, retains the structure of switching between dynamical systems, and covers most situations of interest to control engineers. We also collect all the technical assumptions used in the sequel.

We consider the following CGHDS:

$$
\left[\mathbb{Z}_{+},\left[\left\{\mathbb{R}^{d_{i}}\right\}_{i=0}^{\infty}, \mathbb{R}_{+},\left\{f_{i}\right\}_{i=0}^{\infty}, U\right], \mathbf{A}, \mathbf{G}, V, \mathbf{C}, \mathbf{D}\right] .
$$

Specifically, our discrete state space is $Q=\mathbb{Z}_{+}$. The continuous state space for $x(\cdot)$ is $X=\left\{X_{i}\right\}_{i=0}^{\infty}$, where each $X_{i}$ is a subset of some Euclidean space $\mathbb{R}^{d_{i}}, d_{i} \in \mathbb{Z}_{+}$.

For simplicity of notation, though, the "destination sets" are specified a priori by sets $D_{i} \in \mathrm{D}$ instead of by the collection of set-valued maps F. Also, with no real loss of generality, we consider $U_{i} \equiv U, V_{i} \equiv V, i \in \mathbb{Z}_{+}$to be the sets of continuous and discrete controls, respectively. However, below we do generalize to allow the vector field to depend on the continuous state at the last jump time and add delay operators to autonomous and controlled jumps.

Note that we have specified a priori regions $A_{i}, C_{i}, D_{i} \subset$ $X_{i}, i \in \mathbb{Z}_{+}$. These are the autonomous jump sets, controlled jump sets, and jump destination sets, respectively. For convenience, let $A, C$, and $D$ denote the unions $\bigcup_{i} A_{i} \times\{i\}$, $\bigcup_{i} C_{i} \times\{i\}$, and $\bigcup_{i} D_{i} \times\{i\}, i \in \mathbb{Z}_{+}$, respectively. The dynamics are specified as follows, with delay maps added to allow nonzero jump times:

1) vector fields $f_{i}: X_{i} \times X_{i} \times U \rightarrow \mathbb{R}^{d_{i}}, i \in \mathbb{Z}_{+}$;

2) jump transition maps $G_{i}: A_{i} \times V \rightarrow D$;

3) autonomous transition delay $\Delta_{a, i}: A_{i} \times V \rightarrow \mathbb{R}_{+}$;

4) controlled transition delay $\Delta_{c, i}: C_{i} \times D \rightarrow \mathbb{R}_{+}$.

In shorthand, we may define $G: A \times V \rightarrow D$ in the obvious manner, i.e., $G((x, i), v) \equiv G_{i}(x, v)$, similarly for $\Delta_{a}$ and $\Delta_{c}$. We now place some assumptions on the foregoing.

Assumption 5.1-Model Assumptions: For each $i \in \mathbb{Z}_{+}$, the following hold: $X_{i}$ is the closure of a connected open subset of Euclidean space $\mathbb{R}^{d_{i}}$ and $d_{i} \in \mathbb{Z}_{+}$, with Lipschitz boundary $\partial X_{i} \cdot A_{i}, C_{i}, D_{i} \subset X_{i}$ are closed. In addition, $\partial A_{i}$ is Lipschitz and contains $\partial X_{i}$.

The maps $G, \Delta_{a}$, and $\Delta_{c}$ are bounded uniformly continuous; the vector fields $f_{i}, i \in \mathbb{Z}_{+}$are bounded (uniformly in $i$ ), uniformly Lipschitz continuous in the first argument, and uniformly equi-continuous with respect to the rest. $U, V$ are compact metric spaces. Below, $u(\cdot)$ is a $U$-valued control process, assumed to be measurable.

Assumption 5.2-Jump Set Separation: $d\left(A_{i}, C_{i}\right)>0$ and $\inf _{i \in \mathbb{Z}_{+}} d\left(A_{i}, D_{i}\right)>0, d$ being the appropriate Euclidean distance.

Assumption 5.3-A Transversality: For each $i, \partial A_{i}$ is an oriented $C^{1}$-manifold without boundary and at each point $x$ on $\partial A_{i}, f_{i}(x, z, u)$ is "transversal" to $\partial A_{i}$ for all choices of $z, u$. By this we require that 1) the flow lines be transversal in the usual sense ${ }^{5}$ and 2) the vector field does not vanish on $\partial A_{i}$.

Assumption 5.4-C Transversality: Same as Assumption 5.3 but with $C_{i}$ replacing $A_{i}$.

Assumptions 5.2-5.4 are technical assumptions. They may be traded for others as discussed in Section IX. The latter two are the most restrictive. However, in the sequel we construct examples pointing out the necessity of such assumptions or ones like them. Assumptions 5.1 and 5.2 give rise to welldefined dynamics as follows.

- Existence and uniqueness in each constituent system is assured (via assumptions on $f_{i}, u$ ).

- Switching times are well defined (since the $A_{i}$ and $C_{i}$ are closed).

- Autonomous switching times do not accumulate (thanks to Assumption 5.2).

The dynamics of the control system is much the same as for the CGHDS above, except that the delay maps give rise to a sequence of prejump times $\left\{\tau_{i}\right\}$ and another sequence of post-jump times $\left\{\Gamma_{i}\right\}$ satisfying $0=\Gamma_{0} \leq \tau_{1} \leq \Gamma_{1}<\tau_{2} \leq$ $\Gamma_{2}<\cdots \leq \infty$. On each interval $\left[\Gamma_{j-1}, \tau_{j}\right)$ with nonempty interior, $x(\cdot)$ evolves according to $\dot{x}(t)=f_{i}(x(t), y, u(t))$ in some $X_{i}$, and $i \in \mathbb{Z}_{+}$. At the next prejump time (say, $\tau_{j}$ ) it jumps to some $D_{k} \in X_{k}$ according to one of the following two possibilities.

1) $x\left(\tau_{j}\right) \in A_{i}$, in which case it must jump to $x\left(\Gamma_{j}\right)=$ $G_{i}\left(x\left(\tau_{j}\right), v_{j}\right) \in D$ at time $\Gamma_{j}=\tau_{j}+\Delta_{a, i}\left(x\left(\tau_{j}\right), v_{j}\right)$, $v_{j} \in V$ being a control input. We call this phenomenon an autonomous jump.

2) $x\left(\tau_{j}\right) \in C_{i}$ and the controller chooses to-it does not have to-move the trajectory discontinuously to $x\left(\Gamma_{j}\right) \in D$ at time $\Gamma_{j}=\tau_{j}+\Delta_{c, i}\left(x\left(\tau_{j}\right), x\left(\Gamma_{j}\right)\right)$. We call this a controlled (or impulsive) jump.

See Fig. 5. The jumps may be thought of as beginning at time $\tau_{i}$ and ending at time $\Gamma_{i} \geq \tau_{i}$.

For $t \in[0, \infty)$, let $[t]=\max _{j}\left\{\Gamma_{j} \mid \Gamma_{j} \leq t\right\}$. The vector field $\xi(t)$ of (1) is given by

$$
\xi(t)=f_{i}(x(t), x[t], u(t))
$$

where $i$ is such that $x(t), x[t] \in X_{i}$, and $u(\cdot)$ is a $U$-valued control process.

As with the CGHDS, we can explicitly show that the above model captures all identified discrete phenomena arising in hybrid systems and subsumes all reviewed and classified hybrid systems models.

Note: The autonomous version of this model (including no controlled jumps) yields unique trajectories in the case of, for instance, $A_{i}$ closed and $A_{i} \cap D_{i}=\emptyset$; see [1].

\section{B. Costs}

Let $a>0$ be a discount factor. We add to our previous model the following known maps.

1) Running cost $k_{i}: X_{i} \times X_{i} \times U \rightarrow \mathbb{R}_{+}$.

2) Autonomous jump cost or transition cost $c_{a, i}: A_{i} \times V \rightarrow$ $\mathbb{R}_{+}$.

${ }^{5}$ Transversality implies that $\partial A_{i}$ is $\left(d_{i}-1\right)$-dimensional. 
3) Controlled jump cost or impulse cost $c_{c, i}: C_{i} \times D \rightarrow$ $\mathbb{R}_{+}$.

Important Note: As for $\Delta_{c}$ before, we may use the shorthand $c_{c}: C \times D \rightarrow \mathbb{R}_{+}$, defined in the obvious way. However, below we suppress reference to the index state. We do the same with the other maps: $c_{a}(x(t), y) \equiv c_{a, i}(x(t), y)$ and $k(x(t), y, u) \equiv k_{i}(x(t), y, u)$ if $x(t) \in X_{i}$. In such a case, it is equivalent to think of $x(t)$ as a member of the formal union $\left\{X_{i}\right\}_{i=0}^{\infty}$, that is, a point is implicitly specified by both its value and the index $i$ of the set to which it belongs.

That this incurs no loss of generality is justified by the arguments in Section IV-C on viewing switching as a special case of impulses. Thus, to ease notation we use this shorthand throughout for the maps $G, \Delta_{a}, \Delta_{c}, c_{a}, c_{c}$, etc. To alert the reader, such formal unions are denoted using the symbol $\sqcup$.

Thus, autonomous jumps are done at a cost of $c_{a}\left(x\left(\tau_{j}\right), v_{j}\right)$ paid at time $\tau_{j}$ and controlled jumps at a cost of $c_{c}\left(x\left(\tau_{j}\right), x\left(\Gamma_{j}\right)\right)$ paid at time $\tau_{j}$. In addition to the costs associated with the jumps as above, the controller also incurs a running cost of $k(x(t), x[t], u(t))$ per unit time during the intervals $\left[\Gamma_{j-1}, \tau_{j}\right), j \in \mathbb{Z}_{+}$.

Assumption 5.5-Cost Assumptions: The maps $c_{a}$ and $c_{c}$ are bounded uniformly continuous; the $k_{i}$ are uniformly bounded and uniformly equi-continuous.

In addition, for all $i, j \in \mathbb{Z}_{+}, c_{c, i}: C_{i} \times D \rightarrow \mathbb{R}_{+}$satisfies

$$
\begin{array}{r}
c_{c}(x, y) \geq c_{0}>0, \quad \forall x \in C_{i}, y \in D, \\
c_{c}(x, y)<c_{c}(x, z)+e^{-a \Delta_{c}(x, z)} c_{c}(z, y) \\
\forall x \in C_{i}, z \in D \cap C_{j}, y \in D .
\end{array}
$$

Assumption 5.6-No Jumps to Infinity: Each $D_{i}$ is bounded and for each $i$, there exists an integer $N(i)<\infty$ such that for $x \in C_{i}, y \in D_{j}, j>N(i), c_{c}(x, y)>\sup _{z} J(z)$, where $J$ is defined in the next section.

Note that (6) rules out from consideration infinitely many controlled jumps in a finite interval, and (7) rules out the merging of post-jump time of a controlled jump with the prejump time of the next controlled jump. Together, they preclude accumulation of controlled jumps and are the same as those made in conventional impulse control [10]. Assumption 5.6 precludes jumping to infinity directly or because there is an infinite number of systems to which to jump. Again, these assumptions may be traded for others as discussed in Section IX. However, in the sequel we construct examples pointing out the necessity of such assumptions or ones like them.

The total discounted cost is ${ }^{6}$

$$
\begin{aligned}
& \int_{\mathcal{T}} e^{-a t} k(x(t), x[t], u(t)) d t+\sum_{i} e^{-a \sigma_{i}} c_{a}\left(x\left(\sigma_{i}\right), v_{i}\right) \\
& \quad+\sum_{i} e^{-a \zeta_{i}} c_{c}\left(x\left(\zeta_{i}\right), x\left(\zeta_{i}^{\prime}\right)\right)
\end{aligned}
$$

where $\mathcal{T}=\mathbb{R}_{+} \backslash\left(\bigcup_{i}\left[\tau_{i}, \Gamma_{i}\right)\right),\left\{\sigma_{i}\right\}$ (respectively, $\left.\left\{\zeta_{i}\right\}\right)$ are the successive prejump times for autonomous (respectively,

\footnotetext{
${ }^{6}$ We use a discounted cost for technical reasons, i.e., with an infinite horizon one needs a discounted or average cost for finiteness. We did not use an average cost since in hybrid systems one is often keenly interested in the transitory behavior (especially in the discrete transition sequence).
}

controlled) jumps and $\zeta_{j}^{\prime}$ is the post-jump time for the $j$ th controlled jump. The decision or control variables over which (8) is to be minimized are

- the continuous control $u(\cdot)$;

- the discrete controls $\left\{v_{i}\right\}$, exercised at the prejump times of autonomous jumps;

- the prejump or intervention times $\left\{\zeta_{i}\right\}$ of controlled jumps and the associated destinations $\left\{x\left(\zeta_{i}^{\prime}\right)\right\}$.

As for the periods $\left[\tau_{j}, \Gamma_{j}\right)$ with nonempty interior, we shall follow the convention that the system remains frozen during these intervals.

For illustration, we describe a simple system using our formalism.

Example 5.7: Consider again the hysteresis system of Example 3.1. It can be modeled as follows. The state space is $X=\left\{X_{-1}, X_{1}\right\}$, with $X_{-1}=[-\Delta, \infty)$ and $X_{1}=$ $(-\infty, \Delta]$. The continuous dynamics is given by

$$
f_{-1}=u-1, \quad f_{1}=u+1 .
$$

The discrete dynamics is governed by the autonomous jump sets $A_{-1}$ and $A_{1}$ and their associated transitions, which are, respectively

$$
(-\Delta,-1) \mapsto(-\Delta,+1), \quad(+\Delta,+1) \mapsto(+\Delta,-1) .
$$

\section{EXISTENCE OF Optimal CONTROLS}

Let $J(x)$ denote the infimum of (8) over all choices of $u(\cdot),\left\{v_{i}\right\},\left\{\zeta_{i}\right\},\left\{x\left(\zeta_{i}^{\prime}\right)\right\}$ when $x(0)=x$. We have the following theorem.

Theorem 6.1: The optimal cost is finite for any initial condition.

Proof: Let $F, K, Q$ be bounds of the $f_{i}, k_{i}$, and $c_{a}$, respectively. Then, choosing to make no controlled jumps and using arbitrary $u, v$ we have that

$$
\begin{aligned}
J(x) & \leq K \int_{0}^{\infty} e^{-a t} d t+\sum_{i} e^{-a \sigma_{i}} Q \\
& \leq K / a+Q \sum_{i} e^{-a \sigma_{i}} .
\end{aligned}
$$

Let $\beta=\inf _{i \in \mathbb{Z}_{+}} d\left(A_{i}, D_{i}\right)$. Then $\sigma_{i+1}-\sigma_{i} \geq \beta / F$, so the second term is bounded by $Q \sum_{i=1}^{\infty}\left(e^{-a \beta / F}\right)^{i}$, which converges.

The following corollary is immediate from the argument above.

Corollary 6.2: There are only finitely many autonomous jumps in finite time.

To see why an assumption like Assumption 5.2 is necessary for the above results, one needs only to consider the following one-dimensional example.

Example 6.3: Let $X_{i}=[0,2], A_{i}=\{0,2\}$, and $f_{i}(\cdot, \cdot, \cdot)$ $\equiv-1$ for each $i \in \mathbb{Z}_{+}$. Also for each $i$, define $C_{i}=\emptyset$, $D_{i}=1 / i^{2}$, and $G\left(A_{i}, \cdot\right) \equiv\left(1 /(i+1)^{2}, i+1\right)$. Finally, let $\Delta_{a}(\cdot, \cdot) \equiv 0$ and $c_{a}(\cdot, \cdot) \equiv 1$. Starting in $X_{1}$ at $x(0)=1$, we see that

$$
x\left(\sum_{i=1}^{N} \frac{1}{i^{2}}\right)=\frac{1}{(N+1)^{2}} .
$$


Since the sum of inverse squares converges, we will accumulate an infinite number of jumps and infinite cost by time $t=\pi^{2} / 6$.

It is well known that there are examples in $R^{n}$ where an optimal control fails to exist when the control space is not convex (e.g., when it is finite). The hybrid case not only inherits these, but adding switching among continuous spaces may only exacerbate the situation. Next, however, we show that $J(x)$ is attained for all $x$ if we extend the class of admissible $u(\cdot)$ to "relaxed" controls. The relaxed control framework [41] is as follows: We suppose that $U=\mathcal{P}\left(U^{\prime}\right)$, defined as the space of probability measures on a compact space $U^{\prime}$ with the topology of weak convergence [47]. Also

$$
\begin{array}{ll}
f_{i}(x, z, u)=\int f_{i}^{\prime}(x, z, u) u(d y), & i \in \mathbb{Z}_{+} \\
k_{i}(x, z, u)=\int k_{i}^{\prime}(x, z, u) u(d y), & i \in \mathbb{Z}_{+}
\end{array}
$$

for suitable $\left\{f_{i}^{\prime}\right\},\left\{k_{i}^{\prime}\right\}$ satisfying the appropriate continuity/Lipschitz continuity requirements. The relaxed control framework and its implications in control theory are well known, and the reader is referred to [41] for details.

Theorem 6.4: An optimal trajectory exists for any initial condition.

Proof: See the Appendix.

It is easy to see why Theorem 6.4 may fail in absence of Assumption 5.6.

Example 6.5: Suppose, for example, $k_{i}(x, z, u) \equiv \alpha_{i}$ and $c_{a}(x, v) \equiv \beta_{i}$ when $x \in X_{i}, c_{c}(x, y) \equiv \gamma_{i, j}$ when $x \in X_{i}$, $y \in X_{j}$, with $\alpha_{i}, \beta_{i}, \gamma_{i, j}$ strictly decreasing with $i, j$. It is easy to conceive of a situation where the optimal choice would be to "jump to infinity" as fast as you can.

The theorem may also fail in the absence of Assumption 5.3 as the following two-dimensional system shows.

Example 6.6:

$$
\begin{aligned}
\dot{x}_{1}(t) & =1, & x_{1}(0) & =0 \\
\dot{x}_{2}(t) & =u, & x_{2}(0) & =0
\end{aligned}
$$

with $u \in[0,1]$ and cost

$$
\int_{0}^{\infty} e^{-t} \min \left\{\left|x_{1}(t)+x_{2}(t)\right|, 10^{20}\right\} d t
$$

with the provision that the trajectory jumps to $\left[10^{10}, 10^{10}\right]$ on hitting a certain curve $A$. For $A$, consider two possibilities:

1) the line segment $\left\{x_{1}=1,-1 \leq x_{2} \leq 0\right\}$, a $C^{1}$ manifold with boundary;

2) the circle $\left\{\left(x_{1}, x_{2}\right) \mid\left(x_{1}-1\right)^{2}+\left(x_{2}+1\right)^{2}=1\right\}$, a $C^{1}$ manifold without boundary, but the vector field $(1, u)$ with $u=0$ is not transversal to it at $(1,0)$.

It is easy to see that the optimal cost is not attained in either case.

Also, it is not enough that the flow lines for each control be transversal in the usual sense as the following one-dimensional example shows.
Example 6.7: Let $X_{1}=X_{2}=[0, R]$, for arbitrary $R>0$

$$
f_{1}(x, y, u)=-x+u, \quad f_{2}(x, y, u)=0, \quad u \in[-1,0]
$$

with running cost $\min \{K,|x|\}$ and $G_{1}(0, \cdot) \equiv(K, 2)$, where $0 \leq K<R$. Choosing, for example, $R>K>1$, one sees that the optimal cost cannot be attained for any $1 \geq x(0)>0$.

Coming back to the relaxed control framework, say that $u(\cdot)$ is a precise control if $u(\cdot)=\delta_{q(\cdot)}(d y)$ for a measurable $q:[0, \infty) \rightarrow U^{\prime}$ where $\delta_{z}$ denotes the Dirac measure at $z \in U^{\prime}$. Let $M$ denote the set of measures on $[0, T] \times U^{\prime}$ of the form $d t u(t, d y)$, where $u(\cdot)$ is a relaxed control and $M_{0}$ its subset, corresponding to precise controls. It is known that $M_{0}$ is dense in $M$ with respect to the topology of weak convergence [41]. In conjunction with the Assumption 5.4, this allows us to deduce the existence of $\epsilon$-optimal control policies using precise $u(\cdot)$, for every $\epsilon>0$.

Theorem 6.8: Under Assumptions 5.4-5.6, for every $\epsilon>0$ an $\epsilon$-optimal control policy exists wherein $u(\cdot)$ is precise.

Proof: See the Appendix.

Remarks: If $\left\{\bar{f}_{i}(x, z, y) \mid y \in U^{\prime}\right\}$ are convex for each $x$ and $z$, a standard selection theorem [41] allows us to replace $u^{\infty}(\cdot)$ in the proof by a precise control which is optimal. Otherwise, using Caratheodory's theorem (which states that each point in a compact subset of $\mathbb{R}^{n}$ is expressible as a convex combination of at most $n+1$ of its extreme points) and the aforementioned selection theorem, one may suppose that for $t \geq 0$, the support of $u^{\infty}(t)$ consists of at most $d_{i}+$ 1 points when $x(t) \in X_{i}$.

\section{The VAlue FunCtion}

In the foregoing, we had set $[0]=0$ and thus $x[0]=$ $x(0)=x_{0}$. More generally, for $x(0)=x_{0} \in X_{i_{0}}$, we may consider $x[0]=y$ for some $y \in X_{i_{0}}$, making a negligible difference in the foregoing analysis. Let $V(x, y)$ denote the optimal cost corresponding to this initial data. Then in dynamic programming parlance, $(x, y) \mapsto V(x, y)$ defines the "value function" for our control problem.

In view of Assumption 5.3, we can speak of the right side of $\partial A_{i}$ as the side on which $f_{i}(\cdot, \cdot, \cdot)$ is directed toward $\partial A_{i}$, $i \in \mathbb{Z}_{+}$. A similar definition is possible for the right side of $\partial C_{i}$ (in light of Assumption 5.4).

Definition 7.1: Say that $\left(x_{n}, y_{n}\right) \rightarrow\left(x_{\infty}, y_{\infty}\right)$ from the right in $\bigsqcup_{i}\left(X_{i} \times X_{i}\right)$ if $y_{n} \rightarrow y_{\infty}$ and either $x_{n} \rightarrow x_{\infty} \notin$ $\bigsqcup_{i}\left(\partial A_{i} \cup \partial C_{i}\right)$ or $x_{n} \rightarrow x_{\infty} \in \bigsqcup_{i}\left(\partial A_{i} \cup \partial C_{i}\right)$ from the right side. $V$ is said to be continuous from the right if $\left(x_{n}, y_{n}\right) \rightarrow$ $\left(x_{\infty}, y_{\infty}\right)$ from the right implies $V\left(x_{n}, y_{n}\right) \rightarrow V\left(x_{\infty}, y_{\infty}\right)$.

Theorem 7.2: $V$ is continuous from the right.

Proof: See the Appendix.

Corollary 7.3: $V$ is continuous on $\bigsqcup_{i}\left(X_{i} \times X_{i}\right) \backslash\left[\left(\partial A_{i} \cup\right.\right.$ $\left.\left.\partial C_{i}\right) \times X_{i}\right]$.

Again, Example 6.7 shows the necessity of the vector field's not vanishing on $\partial A_{i}$. Unfortunately, $V$ need not be continuous in the data of the hybrid system.

Example 7.4: Let $X_{1}=X_{2}=\mathbb{R}, f_{1}=f_{2} \equiv 1, k_{1}=1$, and $k_{2}=0$. Further, let $A_{1}=\{p\}, G_{1}(p)=(p, 2)$, and 
$c(p, p)=0$. Then we have

$$
V(x, y)= \begin{cases}0, & x \in X_{2} \\ \left(1-e^{-9(p-x)}\right) / 9, & x \in X_{1}, x \leq p \\ 1 / 9, & x \in X_{1}, x>p .\end{cases}
$$

It is clear that the optimal cost-to-go is not continuous at $p$ in the autonomous jump set data $A_{1}$.

We shall now formally derive the GQVI's $V(\cdot, \cdot)$ is expected to satisfy. Let $\mathcal{C}=\bigsqcup_{i}\left(C_{i} \times X_{i}\right)$ and $E \subset \mathcal{C}$ the set on which

$$
V(x, y)=\min _{z \in D}\left\{c_{c}(x, z)+e^{-a \Delta_{c}(x, z)} V(z, z)\right\}
$$

where $i \in \mathbb{Z}_{+}$is such that $x, y \in X_{i}$. For $(x, y) \in E$, if $x(t)=x$ and $x[t]=y$, an optimal decision (not necessarily the only one) would be to jump to a $z$ where the minimum on the right-hand side of (9) is obtained. On the other hand, for $(x, y) \in \mathcal{C} \backslash E$

$$
V(x, y)<\min _{z \in D}\left\{c_{c}(x, z)+e^{-a \Delta_{c}(x, z)} V(z, z)\right\}
$$

with $i$ as above, and it is not optimal to execute a controlled jump. For $x \in A_{i}$, however, an autonomous jump is mandatory and thus

$V(x, y)=\min _{v}\left\{c_{a}(x, v)+e^{-a \Delta_{a}(x, v)} V(G(x, v), G(x, v))\right\}$.

Suppose $E$ is a closed subset of $\bigsqcup_{i}\left(X_{i} \times X_{i}\right)$. Let $H=$ $E \sqcup\left(\bigsqcup_{i}\left(A_{i} \times X_{i}\right)\right)$, with $M=\left(\bigsqcup_{i}\left(X_{i} \times X_{i}\right)\right) \backslash H$. Let $(x, y) \in M^{\circ}$, with $x, y \in X_{i_{0}}$ (say). Let $O$ be a bounded open neighborhood of $(x, y)$ in $M^{o}$ with a smooth boundary $\partial O$ and $\nu=\inf \{t \geq 0 \mid(x(t), y) \notin O\}$, where $x(\cdot)$ satisfies

$$
\dot{x}(t)=f_{i_{0}}(x(t), y, u(t)), \quad x(0)=x, t \in[0, \nu] .
$$

Note that $y$ is a fixed parameter here. By standard dynamic programming arguments, $V(x, y), x \in \bar{O}, y$, as above, is also the value function for the "classical" control problem of controlling $(10)$ on $[0, \nu]$ with cost

$$
\int_{0}^{\nu} e^{-a t} k_{i_{0}}(x(t), y, u(t)) d t+e^{-a \nu} h(x(\nu), y)
$$

where $h(\cdot, \cdot) \equiv V(\cdot, \cdot)$ on $\partial O$. It follows that $V(x, y)$, $(x, y) \in O$ is the viscosity solution of the Hamilton-Jacobi equation for this problem [42], i.e., it must satisfy (in the sense of viscosity solutions) the partial differential equation

$$
\begin{aligned}
& \min _{u}\left\{\left\langle\nabla_{x} V(x, y), f_{i_{0}}(x, y, u)\right\rangle\right. \\
& \left.\quad-a V(x, y)+k_{i_{0}}(x, y, u)\right\}=0
\end{aligned}
$$

in $O$ and, hence, on $M^{\circ}$. (Here $\nabla_{x}$ denotes the gradient in the $x$ variable.) Elsewhere, standard dynamic programming heuristics suggest that (11) holds with "=" replaced by " $\leq$."

Based on the foregoing discussion, we propose the following system of GQVI's for $V(\cdot, \cdot)$ : For $(x, y) \in X_{i} \times X_{i}$

$$
\begin{aligned}
V(x, y) \leq \min _{z \in D}\left\{c_{c}(x, z)+e^{-a \Delta_{c}(x, z)} V(z, z)\right\} \text { on } \mathcal{C} \\
V(x, y) \leq \min _{v}\left\{c_{a}(x, v)+e^{-a \Delta_{a}(x, v)}\right. \\
\cdot V(G(x, v), G(x, v))\} \text { on } \bigsqcup_{i}\left(A_{i} \times X_{i}\right)
\end{aligned}
$$

$$
\begin{gathered}
\min _{u}\left\{\left\langle\nabla_{x} V(x, y), f_{i}(x, y, u)\right\rangle-a V(x, y)\right. \\
\left.\quad+k_{i}(x, y, u)\right\} \leq 0 \\
\left(V(x, y)-\min _{z \in D}\left\{c_{c}(x, z)+e^{-a \Delta_{c}(x, z)} V(z, z)\right\}\right) \\
\cdot\left(\operatorname { m i n } _ { u } \left\{\left\langle\nabla_{x} V(x, y), f_{i}(x, y, u)\right\rangle-a V(x, y)\right.\right. \\
\left.\left.+k_{i}(x, y, u)\right\}\right)=0 \text { on } \mathcal{C} .
\end{gathered}
$$

Equation (15) states that at least one of (12) or (14) must be an equality on $\mathcal{C}$. Equations (12)-(15) generalize the traditional quasi-variational inequalities encountered in impulse control [10]. We do not address the issue of well-posedness of (12)-(15).

The following "verification theorem," however, can be proved by routine arguments.

Theorem 7.5: Suppose (12)-(15) has a "classical" solution $V$ which is continuously differentiable "from the right" in the first argument and continuous in the second. Suppose $x(\cdot)$ is an admissible trajectory of our control system with initial data $\left(x_{0}, y_{0}\right)$ and $u(\cdot),\left\{v_{i}\right\},\left\{\sigma_{i}\right\},\left\{\zeta_{i}\right\},\left\{\tau_{i}\right\},\left\{\Gamma_{i}\right\}$ the associated controls and jump times, such that the following hold:

1) for a.e. $t \in \mathcal{T}, i$ such that $x(t) \in X_{i}$

$$
\begin{aligned}
& \left\langle\nabla_{x} V(x(t), x[t]), f_{i}(x(t), x[t], u(t))\right\rangle+k_{i}(x(t), x[t], u(t)) \\
& =\min _{u}\left\{\left\langle\nabla_{x} V(x(t), x[t]), f_{i}(x(t), x[t], u)\right\rangle\right. \\
& \left.\quad+k_{i}(x(t), x[t], u)\right\} ;
\end{aligned}
$$

2) for all $i$

$$
\begin{aligned}
V\left(x\left(\sigma_{i}\right), x\left[\sigma_{i}\right]\right)= & c_{a}\left(x\left(\sigma_{i}\right), v_{i}\right)+\exp \left\{-a \Delta_{a}\left(x\left(\sigma_{i}\right), v_{i}\right)\right\} \\
& \cdot V\left(G\left(x\left(\sigma_{i}\right), v_{i}\right), G\left(x\left(\sigma_{i}\right), v_{i}\right)\right)
\end{aligned}
$$

3) for all $i$

$$
\begin{aligned}
V\left(x\left(\zeta_{i}\right), x\left[\zeta_{i}\right]\right)= & c_{c}\left(x\left(\zeta_{i}\right), x\left(\zeta_{i}^{\prime}\right)\right)+\exp \left\{-a \Delta_{c}\left(x, x\left(\zeta_{i}^{\prime}\right)\right)\right\} \\
& \cdot V\left(x\left(\zeta_{i}^{\prime}\right), x\left(\zeta_{i}^{\prime}\right)\right) .
\end{aligned}
$$

Then $x(\cdot)$ is an optimal trajectory.

\section{EXAMPLE PROBLEMS}

Here, we consider some example problems in our framework.

Example 8.1: Consider Example 6.7 except with the controls restricted in $[-1,-\epsilon], 0<\epsilon<0$. Then, the flows are transversal and do not vanish on $A_{1}=\{0\}$ for any $u$. In this case, the optimal control exists. For example, if $K>1 / \epsilon$, one can show that $u(\cdot) \equiv-\epsilon$ is optimal for small enough $x$.

More interestingly, consider the system of Example 3.1. As a control problem, consider minimizing

$$
J=\int_{0}^{\infty} \frac{1}{2}\left(q x^{2}+u^{2}\right) e^{-a t} d t \equiv \int_{0}^{\infty} k(x, u) e^{-a t} d t .
$$

We first solve for $V(x, s=H(x))$ and then $u$. By symmetry, we expect $V(-\Delta, 1)=V(\Delta,-1)$. From the GQVI's, we 
expect $V$ to satisfy

$$
\begin{aligned}
& \min _{u}\left\{-a V(x, s)+V_{x}(x, s) \cdot f(x, u)+k(x, u)\right\}=0 \\
& V(\Delta, 1)=c+V(\Delta,-1) \\
& V(-\Delta,-1)=c+V(-\Delta, 1)
\end{aligned}
$$

where $s$ can take on the values \pm 1 and $c$ represents the cost associated with the autonomous switchings.

We have solved these equations numerically using the algorithms summarized in Section IX-B. As the state is increasingly penalized, the control action increases in such a way to "invert" the hysteresis function $H$. See [1] for more details and other examples.

\section{CONClusions AND Discussion}

We examined the phenomena that arise in hybrid systems and classified several hybrid systems models from the literature. We then proposed a very general mathematical model for hybrid control problems that encompasses these hybrid phenomena and all reviewed models. An optimal control problem was then formulated, studied, and solved in this framework, leading to an existence result for optimal controls. The "value function" associated with this problem is expected to satisfy a set of "GQVI's." Therefore, the foregoing represents initial steps toward developing a unified "statespace" paradigm for hybrid control.

\section{A. Open Issues}

Several open issues suggest themselves. Below is a brief list of some of the more striking ones.

1) A daunting problem is to characterize the value function as the unique viscosity solution of the GQVI's (12)-(15). As mentioned in Section II, following [22], it appears promising.

2) Many of our assumptions can possibly be relaxed at the expense of additional technicalities or traded for alternative sets of assumptions that have the same effect. For example, the condition $d\left(C_{i}, A_{i}\right)>0$ could be dropped by having $c_{c}$ penalize highly the controlled jumps that take place too close to $A_{i}$. (In this case, Assumption 5.4 has to be appropriately reformulated.)

3) Example 6.6 show that Assumption 5.3 cannot be dropped. In the autonomous case, however, the set of initial conditions that hit a $C^{\infty}$ manifold are of measure zero [16]. Thus, one might hope that an optimal control would exist for almost all initial conditions in the absence of Assumption 5.3. The system of Example 6.7 showed this to be false. Likewise, in the systems of Example 6.6 we have, respectively, no optimal control for the sets

$$
\begin{aligned}
& \left\{\left(x_{1}, x_{2}\right) \mid x_{2} \leq 0, x_{1}<1, x_{2}+1>x_{1}\right\} \\
& \left\{\left(x_{1}, x_{2}\right) \mid x_{2} \leq 0, x_{1}<a, x_{2}+a>x_{1}\right\} \\
& \quad \cdot \cup\left([0,1] \times[-a / 2,0]-\overline{B\left([1,-1]^{T}, 1\right)}\right)
\end{aligned}
$$

where $a=2-\sqrt{2}$ and $B(x, r)$ denotes the ball of radius $r$ about the point $x$.
It remains open how to relax Assumptions 5.3 and 5.4. This might be accomplished through additional continuity assumptions on $G, \Delta_{a}$, and $c_{a}$.

4) Another possible extension is in the direction of replacing $X_{i_{0}}$ by smooth manifolds with boundary embedded in a Euclidean space; see [43] for some related work.

5) In light of Definition 7.1, all the proofs seem to hold if Assumption 5.2 is relaxed to only consider distances "from the right," that is, if $\inf _{i} d_{+}\left(A_{i}, D_{i}\right)>0$, with

$$
d_{+}\left(A_{i}, D_{i}\right) \equiv \inf _{t>0, u(\cdot), x \in D_{i}} E_{i}^{t}(x, u(\cdot)) \in A_{i}
$$

where $E_{i}^{t}(x, u(\cdot))$ denotes the solutions under $f_{i}$ with initial condition $x$ and control $u(\cdot)$ in $U^{[0, t)}$. Here, time can be used as a "distance" in light of the uniform bound on $f_{i}$; we consider $t>0$ by adding the caveat that if we jump directly onto $A_{i}$, we do not make another jump until we hit it again. Presumably one must also make some transversality or continuity assumptions for well-posedness. This would allow the results to extend to many more phenomena, including those examples in [43].

6) Another interesting avenue is to study the case where there is an output map, and control actions must be chosen based only on this indirect observation of the full state.

\section{B. Algorithms}

An important issue is to develop good computational schemes to compute near-optimal controls. This is a daunting problem in general as the aforementioned results of [9] show that even smooth Lipschitz differential equations can simulate arbitrary Turing machines, with state dimension as small as three. Thus, it is not hard to conceive of (low-dimensional) control problems where the cost is less than one if the corresponding TM does not halt, but is greater than three if it does. Allowing the possibility of a controlled jump at the initial condition that would result in a cost of two, one sees that finding the optimal control is equivalent to solving the halting problem.

However, in other work, Branicky and Mitter have outlined four approaches to solving the GQVI's associated with optimal hybrid control problems; see [1] and [44] for details, which will be published in full as a companion to this paper.

The first approach solves the GQVI's directly as a boundaryvalue problem; iterations that build on traditional solution techniques in each of the constituent dynamical systems can be devised. More generally, such successive iterative techniques may be used to break complexity by solving hybrid control problems hierarchically; i.e., solve the constituent problems separately, update boundary values due to autonomous- and controlled-switching sets, then repeat.

A stronger algorithmic basis for solving these GQVI's is the following generalized Bellman equation:

$$
V^{*}(x)=\min _{p \in \Pi}\left\{g(x, p)+V^{*}\left(x^{\prime}(x, p)\right)\right\}
$$

where $\Pi$ is a generalized set of actions, $g$ measures incremental cost of action $p$ from state $x$, and $x^{\prime}$ is the resulting state when 
$p$ is applied from $x$. The three classes of actions available in our hybrid systems framework at each $x$ are as follows:

- Continuous Controls: $u \in U$;

- Controlled Jumps: choosing source and destination (if $x \in C$ );

- Autonomous Jumps: possibly modulated by discrete controls $v \in V$ (if $x \in A$ ).

From this viewpoint, generalized policy and value iteration become solution tools.

One key to efficient algorithms for solving optimal hybrid control problems lies in noticing their strong connection to the models of impulse control and piecewise-deterministic processes [18], [19]. Making this explicit, we have developed algorithms similar to those for impulse control and one based on linear programming. The latter seems more promising. While the resulting linear programs are large-due to the curse of dimensionality - they are sparse. Further, they have a special structure both as linear programs and due to the hierarchical nature of discrete versus continuous states of hybrid systems. Exploiting this structure is a topic of current research; see [45] for work relating to the hierarchical structure of discrete and hybrid systems.

Finally, although solving these inequalities has high computational complexity, in general-if they are solvable at all-they are no more complex than synthesis methods for general nonlinear systems. Thus, a current research goal is to isolate those problems (such as linear constituent dynamics, polyhedral jump sets, and quadratic costs) that lead to tractable solutions (such as small quadratic or even linear programs).

\section{APPENDIX}

Proof of Remark 4.1: First, we show that autonomous switching can be viewed as a special case of autonomous impulses by embedding the discrete states into a larger continuous-state space. Consider the differential equation with parameters $\dot{x}=f(x, p)$, where $x \in \mathbb{R}^{n}, p \in P \subset \mathbb{R}^{m}$ closed, and $f: \mathbb{R}^{n} \times P \rightarrow \mathbb{R}^{n}$ continuous. Let $\nu: \mathbb{R}^{n} \times P \rightarrow P$ be the function governing autonomous switching. (For example, in the Tavernini model, $\nu$ is the "discrete dynamics.")

Then, since $\mathbb{R}^{n}$ has the universal extension property [46], we can extend $f$ to a continuous function $F: \mathbb{R}^{n} \times \mathbb{R}^{m} \rightarrow \mathbb{R}^{n}$. Now, consider the ODE on $\mathbb{R}^{n+m}$

$$
\dot{x}=F(x, \xi), \quad \dot{\xi}=0
$$

where $x \in \mathbb{R}^{n}, \xi \in \mathbb{R}^{m}$, and $F: \mathbb{R}^{n} \times \mathbb{R}^{m} \rightarrow \mathbb{R}^{n}$ continuous. Let the transition function be $G: \mathbb{R}^{n} \times P \rightarrow$ $\mathbb{R}^{n} \times P$ with $G(x, p)=(x, \nu(x, p))$.

Now, we show that continuous switching can be viewed as a special case of continuous impulses. A system with controlled switching is described by

$$
\dot{x}(t)=f(x(t), u(t)), \quad x(0)=x_{0} \in \mathbb{R}^{d}
$$

where $u(\cdot)$ is a piecewise constant function taking values in $U \subset \mathbb{R}^{m}$ and $f: \mathbb{R}^{d} \times U \rightarrow \mathbb{R}^{d}$ is a map with sufficient regularity. There is a strictly positive cost associated with the switchings of $u(\cdot)$. In our framework, let $x^{\prime}(\cdot)=[x(\cdot), u(\cdot)]^{T}$ be the new state process with dynamics

$$
\dot{x}^{\prime}(t)=f^{\prime}\left(x^{\prime}(t)\right), \quad f^{\prime}(\cdot)=[f(\cdot), 0]^{T}
$$

taking values in $X=\left\{X_{i}\right\}_{i=0}^{\infty}$ where each $X_{i}$ is a copy of $\mathbb{R}^{d} \times U$. Set $C_{i}=X_{i}, F_{i} \equiv\left\{X_{i}\right\}, A_{i}=\emptyset$ for $i \in \mathbb{Z}_{+}$. Switchings of $u(\cdot)$ now correspond to controlled jumps with the associated costs.

Proof of Theorem 6.4: Fix $x(0)=x_{0} \in X_{i_{0}}, i_{0} \in \mathbb{Z}_{+}$. Consider a sequence

$$
\begin{aligned}
& \left(x^{n}(\cdot), u^{n}(\cdot),\left\{v_{i}^{n}\right\},\left\{\sigma_{i}^{n}\right\},\left\{\zeta_{i}^{n}\right\},\left\{\tau_{i}^{n}\right\},\left\{\Gamma_{i}^{n}\right\}\right), \\
& \quad n \in \mathbb{Z}_{+}
\end{aligned}
$$

associated with our control system, with the obvious interpretation, such that $x^{n}(0)=x_{0}$ for all $n$ and the corresponding costs decrease to $J\left(x_{0}\right)$. Let $y^{n}(\cdot)$ denote the solution of

$$
\dot{y}^{n}(t)=f_{i_{0}}\left(y^{n}(t), x_{0}, u^{n}(t)\right), \quad y^{n}(0)=x_{0}, n \in \mathbb{Z}_{+} .
$$

Then $x^{n}(\cdot)$ and $y^{n}(\cdot)$ agree on $\left[0, \tau_{1}^{n}\right)$. Since $\left\{f_{i}\right\}$ are bounded, $\left\{y^{n}(\cdot)\right\}$ are equi-continuous bounded in $C\left(\mathbb{R}_{+} ; \mathbb{R}^{d_{i_{0}}}\right)$, hence relatively sequentially compact by the Arzela-Ascoli theorem. The finite nonnegative measures $\eta^{n}(d t, d y)=d t u^{n}(t, d y)$ on $[0, T] \times U^{\prime}$ are relatively sequentially compact in the topology of weak convergence by Prohorov's theorem [47]. $\left\{\tau_{1}^{n}\right\}$ are trivially relatively compact in $[0, \infty]$. Thus dropping to a subsequence if necessary, we may suppose that $y^{n}(\cdot) \rightarrow y^{\infty}(\cdot), \eta^{n}(d t, d y) \rightarrow \eta^{\infty}(d t, d y)$, $\tau_{1}^{n} \rightarrow \tau_{1}^{\infty}$ in the respective spaces. Clearly $\eta^{\infty}$ disintegrates as $\eta^{\infty}(d t, d y)=d t u^{\infty}(t, d y)$. Rewrite (18) as

$$
\begin{aligned}
y^{n}(t)= & x_{0}+ \\
& \left(\int_{0}^{t} f_{i_{0}}\left(y^{n}(s), x_{0}, u^{n}(s)\right)\right. \\
& \left.\quad-f_{i_{0}}\left(y^{\infty}(s), x_{0}, u^{n}(s)\right) d s\right) \\
+ & \int_{0}^{t} f_{i_{0}}\left(y^{\infty}(s), x_{0}, u^{n}(s)\right) d s
\end{aligned}
$$

for $t \geq 0$. By the uniform Lipschitz continuity of $f_{i_{0}}$, the term in parentheses tends to zero as $n \rightarrow \infty$. Since $\eta^{n} \rightarrow \eta^{\infty}$, the last term, in view of the relaxed control framework, converges to

$$
\int_{0}^{t} f_{i_{0}}\left(y^{\infty}(s), x_{0}, u^{\infty}(s)\right) d s
$$

for $t \in[0, T]$. Since $T$ was arbitrary, a standard argument allows us to extend this claim to $t \in[0, \infty)$. (We use [47, Th. 2.1(v), p. 12] and the fact that $\eta^{\infty}\left(\{t\} \times U^{\prime}\right)=0$.) Hence, $y^{\infty}(\cdot)$ and $u^{\infty}(\cdot)$ satisfy (18) with $n=\infty$. Since $d\left(C_{i_{0}}, A_{i_{0}}\right)>0$, either $\tau_{i}^{n}=\sigma_{i}^{n}$ for sufficiently large $n$, or $\tau_{i}^{n}=\zeta_{i}^{n}$ for sufficiently large $n$. Suppose the first possibility holds. Then $y^{\infty}\left(\tau_{1}^{\infty}\right)=\lim x^{n}\left(\tau_{1}^{n}\right) \in A_{i_{0}}$. Let $v_{i}^{n} \rightarrow v_{i}^{\infty}$ in $V$ along a subsequence. Then $c_{a}\left(x^{n}\left(\tau_{1}^{n}\right), v_{1}^{n}\right) \rightarrow$ $c_{a}\left(y^{\infty}\left(\tau_{1}^{\infty}\right), v_{1}^{\infty}\right), \Delta_{a}\left(x^{n}\left(\tau_{1}^{n}\right), v_{1}^{n}\right) \rightarrow \Delta_{a}\left(y^{\infty}\left(\tau_{1}^{\infty}\right), v_{1}^{\infty}\right)$, $\Gamma_{1}^{n} \rightarrow \Gamma_{1}^{\infty} \equiv \tau_{1}^{\infty}+\Delta_{a}\left(y^{\infty}\left(\tau_{1}^{\infty}\right), v_{1}^{\infty}\right)$. Set $x^{\infty}(\cdot)=y^{\infty}(\cdot)$ on $\left[0, \tau_{1}^{\infty}\right]$ and $x^{\infty}\left(\Gamma_{1}^{\infty}\right)=G\left(x^{\infty}\left(\tau_{1}^{\infty}\right), v_{1}^{\infty}\right)$. Then

$$
\begin{gathered}
\int_{0}^{\tau_{1}^{\tau_{1}}} e^{-a t} k_{i_{0}}\left(x^{n}(t), x_{0}, u^{n}(t)\right) d t \rightarrow \\
\int_{0}^{\tau_{1}^{\infty}} e^{-a t} k_{i_{0}}\left(x^{\infty}(t), x_{0}, u^{\infty}(t)\right) d t .
\end{gathered}
$$


If the second possibility holds instead, one similarly has $y_{1}\left(\tau_{1}^{\infty}\right) \in C_{i_{0}}$. Then Assumption 5.6 ensures that $\left\{x^{n}\left(\Gamma_{1}^{n}\right)\right\}$ is a bounded sequence in $D$ and hence converges along a subsequence to some $y^{\prime} \in D$. Then, on dropping to a further subsequence if necessary, $c_{c}\left(x^{n}\left(\tau_{1}^{n}\right), x^{n}\left(\Gamma_{1}^{n}\right)\right) \rightarrow c_{c}\left(y^{\infty}\left(\tau_{1}^{n}\right), y^{\prime}\right)$, $\Delta_{c}\left(x^{n}\left(\tau_{1}^{n}\right), x^{n}\left(\Gamma_{1}^{n}\right)\right) \rightarrow \Delta_{c}\left(y^{\infty}\left(\tau_{1}^{n}\right), y^{\prime}\right)$. Set $x^{\infty}(\cdot)=y^{\infty}(\cdot)$ on $\left[0, \tau_{1}^{\infty}\right], \Gamma_{1}^{\infty}=\tau_{1}^{\infty}+\Delta_{c}\left(y^{\infty}\left(\tau_{1}^{\infty}\right), y^{\prime}\right)=\lim \Gamma_{1}^{n}$ and $x^{\infty}\left(\Gamma_{1}^{\infty}\right)=y^{\prime}$. Again (19) holds. Note that in both cases, $x^{\infty}(\cdot)$ defined on $\left[0, \tau_{1}^{\infty}\right]$ is an admissible segment of a controlled trajectory for our system. The only way it would fail to be so is if it hit $A_{i_{0}}$ in $\left[0, \tau_{1}^{\infty}\right)$. If so, $x^{n}(\cdot)$ would have to hit $A_{i_{0}}$ in $\left[0, \tau_{1}^{n}\right)$ for sufficiently large $n$ by virtue of Assumption 5.3, a contradiction.

Now repeat this argument for $\left\{x^{n}\left(\Gamma_{1}^{\infty}+\cdot\right)\right\}$ in place of $\left\{x^{n}(\cdot)\right\}$. The only difference is a varying but convergent initial condition instead of a fixed one, which causes only minor alterations in the proof. Iterating, one obtains an admissible trajectory $x^{\infty}(\cdot)$ with cost $J\left(x_{0}\right)$.

Proof of Theorem 6.8: Recall the setup of Theorem 6.4. Consider the time interval $\left[0, \tau_{1}^{\infty}\right]$. Let $\bar{u}^{n}(\cdot)$ and $n \in \mathbb{Z}_{+}$ be precise controls such that $d t \bar{u}^{n}(t, d y) \rightarrow \eta^{\infty}(d t, d y)=$ $d t u^{\infty}(t, d y)$ in the topology of weak convergence. Let $\bar{y}^{n}(\cdot)$ and $n \in \mathbb{Z}_{+}$denote the corresponding solutions to (18). Now $\tau_{1}^{\infty}$ equals either $\sigma_{1}^{\infty}$ or $\zeta_{1}^{\infty}$. Suppose the former holds. As in the proof of Theorem 6.4 , we have $\bar{y}^{n} \rightarrow y^{\infty}(\cdot)$ in $C\left([0, \infty), X_{i_{0}}\right)$. Using Assumption 5.3 as in the proof of Theorem 6.4, one verifies that

$$
\bar{\sigma}_{1}^{n} \equiv \inf \left\{t \geq 0 \mid \bar{y}^{n}(t) \in A_{i_{0}}\right\} \rightarrow \sigma_{1}^{\infty} .
$$

Thus for any $\delta>0$, we can take $n$ large enough such that

$$
\begin{aligned}
& \left|\sigma_{1}^{\infty}-\bar{\sigma}_{1}^{n}\right|<\delta \\
& \sup \left\{\left\|\bar{y}^{n}(t)-y^{\infty}(t)\right\| \text { s.t. } 0 \leq t<\sigma_{1}^{\infty} \vee \bar{\sigma}_{1}^{n}\right\}<\delta \\
& \left|\bar{\sigma}_{1}^{n}+\Delta_{a}\left(\bar{y}^{n}\left(\bar{\sigma}_{1}^{n}\right), v_{1}^{\infty}\right)-\Gamma_{1}^{\infty}\right|<\delta .
\end{aligned}
$$

Set $\bar{x}^{n}(\cdot)=\bar{y}^{n}(\cdot)$ on $\left[0, \bar{\sigma}_{1}^{n}\right]$ and $\bar{x}^{n}\left(\bar{\sigma}^{n}+\Delta_{a}\left(\bar{x}^{n}\left(\bar{\sigma}_{1}^{n}\right), v_{1}^{\infty}\right)\right)$ $=G\left(\bar{x}^{n}\left(\bar{\sigma}_{1}^{n}\right), v_{1}^{\infty}\right)$ (corresponding to control action $v_{1}^{\infty}$ ). The latter may be taken to lie in the open $\delta$-neighborhood of $x^{\infty}\left(\Gamma_{1}^{\infty}\right)$ by further increasing $n$ if necessary. In case $\tau_{1}^{\infty}=\zeta_{1}^{\infty}$, one uses Assumption 5.4 instead to conclude that $\bar{y}^{n}\left(t_{n}^{\prime}\right) \in C_{i_{0}}$ for some $t_{n}^{\prime}$ in the $\delta$-neighborhood of $\tau_{1}^{\infty}$ for $n$ sufficiently large. Set $\bar{\zeta}_{1}^{n}=t_{n}^{\prime}, \bar{x}^{n}(\cdot)=\bar{y}^{n}(\cdot)$ on $\left[0, \bar{\zeta}^{n}\right)$. By further increasing $n$ if necessary, we may also ensure that

$$
\begin{array}{r}
\left\{\bar{x}^{n}(t) \mid t \in\left[0, \bar{\zeta}^{n}\right)\right\} \cap A_{i_{0}}=\emptyset \\
\sup \left\{\| \bar{y}^{n}(t)-x^{\infty}(t)|| \mid 0 \leq t \leq \bar{\zeta}_{1}^{n} \wedge \bar{\zeta}_{1}^{\infty}\right\}<\delta \\
\left|\bar{\zeta}_{1}^{n}+\Delta_{c}\left(\bar{x}^{n}\left(\bar{\zeta}_{1}^{n}\right), x^{\infty}\left(\Gamma_{1}^{\infty}\right)\right)-\Gamma_{1}^{\infty}\right|<\delta .
\end{array}
$$

Set

$$
\bar{x}^{n}\left(\bar{\zeta}_{1}^{n}+\Delta_{c}\left(\bar{x}^{n}\left(\bar{\zeta}_{1}^{n}\right), x^{\infty}\left(\Gamma_{1}^{\infty}\right)\right)\right)=x^{\infty}\left(\Gamma_{1}^{\infty}\right) .
$$

It is clear how to repeat the above procedure on each interval between successive jump times to construct an admissible trajectory $\bar{x}^{n}(\cdot)$ with cost within $\epsilon$ of $J\left(x_{0}\right)$ for a given $\epsilon$ $>0$.
Proof of Theorem 7.2: Let $\left(x_{n}, y_{n}\right) \rightarrow\left(x_{\infty}, y_{\infty}\right)$ from the right in $\bigsqcup_{i}\left(X_{i} \times X_{i}\right)$ and let $\bar{x}^{n}(\cdot), n \in \mathbb{Z}_{+} \cup\{\infty\}$ denote optimal trajectories for initial data $\left(x_{n}, y_{n}\right)$, respectively. By dropping to a subsequence of $n \in \mathbb{Z}_{+}$if necessary, obtain as in Theorem 6.4 a limiting admissible trajectory $x^{\prime}(\cdot)$ for initial data $\left(x_{\infty}, y_{\infty}\right)$ with cost (say) $\alpha$ such that $V\left(x_{n}, y_{n}\right) \rightarrow$ $\alpha \geq V\left(x_{\infty}, y_{\infty}\right)$. Suppose $\alpha>V\left(x_{\infty}, y_{\infty}\right)+3 \epsilon$ for some $\epsilon>0$. Starting from $x^{\infty}(\cdot)$, argue as in Theorem 6.8 to construct a trajectory $\tilde{x}^{n}(\cdot)$ with initial data $\left(x_{n}, y_{n}\right)$ for $n$ sufficiently large so that the corresponding cost does not exceed $V\left(x_{\infty}, y_{\infty}\right)+\epsilon$. At the same time, $V\left(x_{n}, y_{n}\right) \geq$ $\alpha-\epsilon>V\left(x_{\infty}, y_{\infty}\right)+2 \epsilon$ for $n$ sufficiently large, which contradicts the fact that $V\left(x_{n}, y_{n}\right)$ is the optimal cost for initial data $\left(x_{n}, y_{n}\right)$. The claim follows.

\section{REFERENCES}

[1] M. S. Branicky, "Studies in hybrid systems: Modeling, analysis, and control," Ph.D. dissertation, Dept. Elec. Eng. and Computer Sci., Massachusetts Inst. Technol., Cambridge, June 1995.

[2] A. Gollu and P. P. Varaiya, "Hybrid dynamical systems," in Proc. IEEE Conf. Decision Contr., Tampa, FL, Dec. 1989, pp. 2708-2712.

[3] R. W. Brockett, "Hybrid models for motion control systems," in Essays in Control, H. Trentelman and J. C. Willems, Eds. Boston, MA: Birkhäuser, 1993, pp. 29-53.

[4] A. Back, J. Guckenheimer, and M. Myers, "A dynamical simulation facility for hybrid systems," in Hybrid Systems, Lecture Notes in Computer Science, vol. 736, R. L. Grossman, A. Nerode, A. P. Ravn, and H. Rischel, Eds. New York: Springer, 1993, pp. 255-267.

[5] P. P. Varaiya, "Smart cars on smart roads: Problems of control," IEEE Trans. Automat. Contr., vol. 38, pp. 195-207, Feb. 1993.

[6] C. S. Hynes, G. Meyer, E. A. Palmer III, and G. H. Hardy, "Vehicle management for the high speed civil transport," NASA Ames Res. Center, Tech. Rep., 1993, extended abstract.

[7] C. S. Hsu, Cell-to-Cell Mapping. New York: Springer, 1987.

[8] S. Di Gennaro, C. Horn, S. R. Kulkarni, and P. J. Ramadge, "Reduction of timed hybrid systems," in Proc. IEEE Conf. Decision Contr., Lake Buena Vista, FL, Dec. 1994, pp. 4215-4220.

[9] M. S. Branicky, "Universal computation and other capabilities of hybrid and continuous dynamical systems," Theoretical Computer Science, vol. 138 , no. 1 , pp. $67-100,1995$

[10] A. Bensoussan and J.-L. Lions, Impulse Control and Quasi-Variational Inequalities. Paris: Gauthier-Villars, 1984.

[11] S. K. Mitter and V. S. Borkar, "6.291; Special topics: Optimal control," Course Notes, Dept. Elec. Eng. Computer Sci., Massachusetts Inst. Technol., Cambridge, Fall 1993.

[12] M. S. Branicky, V. Borkar, and S. K. Mitter, "A unified framework for hybrid control," Laboratory for Information and Decision Systems, Massachusetts Inst. Technol., Cambridge, Tech. Rep. LIDS-P-2239, Apr. 1994.

[13] __ "A unified framework for hybrid control," in Proc. 11th INRIA Int. Conf. Anal. Optim. Syst., Lecture Notes in Control and Information Sciences, vol. 199, G. Cohen and J.-P. Quadrat, Eds. New York: Springer, 1994, pp. 352-358.

[14] _ "A unified framework for hybrid control," in Proc. IEEE Conf. Decision Contr., Lake Buena Vista, FL, Dec. 1994, pp. 4228-4234.

[15] H. S. Witsenhausen, "A class of hybrid-state continuous-time dynamic systems," IEEE Trans. Automat. Contr., vol. 11, pp. 161-167, Feb. 1966.

[16] L. Tavernini, "Differential automata and their discrete simulators," Nonlinear Anal., Theory, Methods, Appl., vol. 11, no. 6, pp. 665-683, 1987.

[17] A. Nerode and W. Kohn, "Models for hybrid systems: Automata, topologies, stability," in Hybrid Systems, Lecture Notes in Computer Science, vol. 736, R. L. Grossman, A. Nerode, A. P. Ravn, and H. Rischel, Eds. New York: Springer, 1993, pp. 317-356.

[18] O. L. V. Costa, "Impulse control of piecewise-deterministic processes via linear programming," IEEE Trans. Automat. Contr., vol. 36, pp. 371-375, Mar. 1991.

[19] O. L. V. Costa and M. H. A. Davis, "Impulse control of piecewise deterministic processes," Math. Contr. Signals Syst., vol. 2, pp. 187-206, 1989. 
[20] M. H. A. Davis, Markov Models and Optimization. London, U.K.: Chapman and Hall, 1993.

[21] J. Zabczyk, "Optimal control by means of switching," Studia Mathematica, vol. 65, pp. 161-171, 1973.

[22] J. Yong, "Systems governed by ordinary differential equations with continuous, switching and impulse controls," Appl. Math. Optim., vol. 20, pp. 223-235, 1989

[23] J. Lu, L. Liao, A. Nerode, and J. H. Taylor, "Optimal control of systems with continuous and discrete states," in Proc. IEEE Conf. Decision Contr., San Antonio, TX, Dec. 1993, pp. 2292-2297.

[24] X. Ge, W. Kohn, and A. Nerode, "Algorithms for chattering approximations to relaxed optimal controls," Math. Sci. Inst., Cornell Univ., Tech. Rep. 94-23, Apr. 1994.

[25] A. Deshpande and P. Varaiya, "Viable control of hybrid systems," in Hybrid Systems II, Lecture Notes in Computer Science, P. Anstaklis, W. Kohn, A. Nerode, and S. Sastry, Eds. New York: Springer, 1995.

[26] W. Kohn, A. Nerode, J. Remmel, and A. Yaknis, "Viability in hybrid systems," Theoretical Computer Sci., vol. 138, no. 1, 1995.

[27] D. D. Bainov and P. S. Simeonov, Systems with Impulse Effect. Chichester, U.K.: Ellis Horwood, 1989.

[28] P. J. Antsaklis, J. A. Stiver, and M. D. Lemmon, "Hybrid system modeling and autonomous control systems," in Hybrid Systems, vol. 736, Lecture Notes in Computer Science, R. L. Grossman, A. Nerode, A. P. Ravn, and H. Rischel, Eds. New York: Springer, 1993, pp. 366-392.

[29] V. I. Arnold, Ordinary Differential Equations. Cambridge, MA: MIT Press, 1973.

[30] J. Guckenheimer and P. Holmes, Nonlinear Oscillations, Dynamical Systems, and Bifurcations of Vector Fields, 3rd printing. New York: Springer, 1990.

[31] M. W. Hirsch and S. Smale, Differential Equations, Dynamical Systems, and Linear Algebra. San Diego, CA: Academic, 1974.

[32] D. G. Luenberger, Introduction to Dynamic Systems. New York: Wiley, 1979.

[33] L. Padulo and M. A. Arbib, System Theory. Philadelphia, PA: W. B. Saunders, 1974.

[34] E. D. Sontag, Mathematical Control Theory. New York: Springer, 1990.

[35] D. Harel, "Statecharts: A visual formalism for complex systems," Sci. Computer Programming, vol. 8, pp. 231-274, 1987.

[36] R. L. Grossman, A. Nerode, A. P. Ravn, and H. Rischel, Eds., Hybrid Systems, Lecture Notes in Computer Science, vol. 736. New York: Springer, 1993.

[37] L. Blum, M. Shub, and S. Smale, "On a theory of computation and complexity over the real numbers," Bull. Amer. Math. Soc., vol. 21, pp. $1-46$, July 1989

[38] G. Meyer, "Design of flight vehicle management systems," in Proc. IEEE Conf. Decision Contr.,, Lake Buena Vista, FL, Plenary lecture, Dec. 1994.

[39] A. Deshpande, "Control of hybrid systems," Ph.D. dissertation, Univ. California, Berkeley, 1994.

[40] J. G. Hocking and G. S. Young, Topology. New York: Dover, 1988.

[41] L. C. Young, Lectures on the Calculus of Variations and Optimal Control Theory, 2nd ed. New York: Chelsea, 1980.

[42] M. G. Crandall, H. Ishii, and P.-L. Lions, "User's guide to viscosity solutions of second order partial differential equations," Bull. Amer. Math. Soc., vol. 27, no. 1, pp. 1-67, 1992.

[43] R. W. Brockett, "Smooth multimode control systems," in BerkeleyAmes Conf. Nonlinear Problems Contr. Fluid Dynamics, C. Martin, Ed. Brookline, MA: Math.-Sci., 1983, pp. 103-110.

[44] M. S. Branicky and S. K. Mitter, "Algorithms for optimal hybrid control," in Proc. IEEE Conf. Decision Contr., New Orleans, LA, Dec. 1995, pp. 2661-2666.
[45] P. E. Caines and Y.-J. Wei, "The hierarchical lattices of a finite machine," Syst. Contr. Lett., vol. 25, no. 4, pp. 257-263, 1995.

[46] J. R. Munkres, Topology. Englewood Cliffs, NJ: Prentice-Hall, 1975.

[47] P. Billingsley, Convergence of Probability Measures. New York: Wiley, 1968

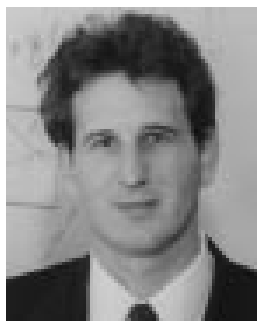

Michael S. Branicky (S'92-M'95) received the B.S. and M.S. degrees in electrical engineering and applied physics from Case Western Reserve University (CWRU) in 1987 and 1990, respectively. $\mathrm{He}$ received the Sc.D. degree in electrical engineering and computer science from the Massachusetts Institute of Technology (MIT), Cambridge, in 1995.

In 1997 he joined CWRU as the Nord Assistant Professor of Engineering. He has held research positions at MIT, Wright-Patterson AFB, NASA Ames, Siemens Corporate Research Center, ARO's Center for Intelligent Control Systems, and Lund Institute of Technology. His research interests include hybrid systems, intelligent control, learning, robotics, and flexible manufacturing.

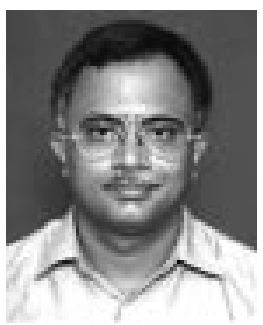

Vivek S. Borkar (SM'95) received the Ph.D. degree from the University of California, Berkeley, in 1980.

He has been a Visiting Scientist at the Massachusetts Institute of Technology Laboratory for Information and Decision Systems, Cambridge, since 1986. He is currently an Associate Professor of Computer Science and Automation at the Indian Institute of Science, Bangalore, India. His research interests include stochastic control, control under communication constraints, hybrid control, stochastic recursive algorithms, neurodynamic programming, and complex adaptive systems.

Dr. Borkar received the IEEE Control Systems Best Transactions Paper Award in 1982, the S. S. Bhatnagar Prize in 1992, and the Homi Bhabha Fellowship for the period 1995-1996.

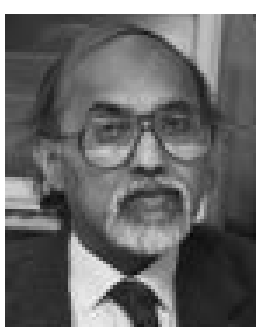

Sanjoy K. Mitter (M'68-SM'77-F'79) received the Ph.D. degree from the Imperial College of Science and Technology, University of London, U.K.

In 1970 he joined the Massachusetts Institute of Technology, Cambridge, where he is currently a Professor of Electrical Engineering and the CoDirector of the Laboratory for Information and Decision systems. He also directs the Center for Intelligent Control Systems, an interuniversity center researching foundations of intelligent systems. His research interests include theory of stochastic dynamical systems; mathematical physics and its relationship to system theory; image analysis and computer vision; and structure, function, and organization of complex systems.

Dr. Mitter was elected to the National Academy of Engineering in 1988. 